\title{
Anisotropy of magnetoviscous effect in structure-forming ferrofluids
}

Article

Accepted Version

Sreekumari, A. and IIg, P. (2015) Anisotropy of magnetoviscous effect in structure-forming ferrofluids. Physical Review E, 92 (1). 012306. ISSN 1539-3755 doi: https://doi.org/10.1103/PhysRevE.92.012306 Available at https://centaur.reading.ac.uk/40567/

It is advisable to refer to the publisher's version if you intend to cite from the work. See Guidance on citing.

Published version at: http://journals.aps.org/pre/abstract/10.1103/PhysRevE.92.012306

To link to this article DOI: http://dx.doi.org/10.1103/PhysRevE.92.012306

Publisher: American Physical Society

All outputs in CentAUR are protected by Intellectual Property Rights law, including copyright law. Copyright and IPR is retained by the creators or other copyright holders. Terms and conditions for use of this material are defined in the End User Agreement.

\section{www.reading.ac.uk/centaur}

\section{CentAUR}

Central Archive at the University of Reading

Reading's research outputs online 


\title{
The anisotropy of magnetoviscous effect in structure-forming ferrofluids
}

\author{
Aparna Sreekumari ${ }^{1}$ and Patrick $\operatorname{Ilg}^{1,2}$ \\ ${ }^{1}$ Polymer Physics, Department of Materials, ETH Zürich, Wolfgang-Pauli Str. 10, CH-8093 Zürich \\ ${ }^{2}$ Department of Mathematics and Statistics, University of Reading, Reading RG6 6AX
}

(Dated: May 22, 2015)

\begin{abstract}
The magnetoviscous effect, change in viscosity with change in magnetic field strength, and the anisotropy of magnetoviscous effect, change in viscosity with orientation of magnetic field, have been a focus of interest since four decades. A satisfactory understanding of the microscopic origin of anisotropy of magnetoviscous effect in magnetic fluids is still a matter of debate and a field of intense research. Here, we present an extensive simulation study to understand the relation between the anisotropy of magnetoviscous effect and the underlying change in micro-structures of ferrofluids. Our results indicate that field-induced chain-like structures respond very differently depending on their orientation relative to the direction of an externally applied shear flow, which leads to a pronounced anisotropy of viscosity. In this work, we focus on three exemplary values of dipolar interaction strengths which correspond to weak, intermediate and strong interactions between dipolar colloidal particles. We compare our simulation results with an experimental study on cobalt-based ferrofluids as well as with an existing theoretical model called the chain model. A non-monotonic behaviour in the anisotropy of magnetoviscous effect is observed with increasing dipolar interaction strength and is explained in terms of micro-structure formation.
\end{abstract}

PACS numbers: 75.50.Mm, 82.20.Wt, 83.80.Hj, 83.80.Gv, 47.32.-y

\section{INTRODUCTION}

Magnetic fluids in general and ferrofluids in particular exhibit interesting viscous properties in the absence and presence of an external field. The viscous properties of ferrofluids have been studied for decades due to the medical and technological applications [1]. The strength of the magnetic field has a major influence on the viscosity of ferrofluids which is known as the magnetoviscous effect (MVE) [1-8]. In addition to the strength of the applied field, its orientation with respect to the flow geometry is found to have an influence on the viscosity of the ferrofluids, which is known as the anisotropy of magnetoviscous effect. It has been first observed in a classic experiment on dilute ferrofluids by McTague in 1968 [9]. In McTague's experiment, an external magnetic field is applied parallel and perpendicular to the capillary tube axis. He found that the viscosity increases when the external magnetic field is applied parallel to the flow direction compared to the change in viscosity when the field is oriented perpendicular to the flow direction. This is due to the occurrence of an additional magnetic torque due to the misalignment of dipole moment and the magnetic field direction. In 1972, Shliomis [10] proposed a theoretical model (NI model) for non-interacting ferrofluid system to understand the anisotropy of MVE. The NI model successfully described the results of McTague's experiment. To observe the viscosity changes in the flow, gradient of the flow and the vorticity directions, Grants et al. [11] carried out experiments in a parallel plate geometry for a weakly interacting system. From this experiment, it is observed that the magnetic field is applied along gradient of the flow direction causes large viscosity than that of the flow and vorticity directions. Later on, a number of experiments have been carried out in a capillary flow ge- ometry in dilute as well as in concentrated ferrofluids [1214]. A clear dependence of MVE on shear rate has been observed experimentally in ferrofluids with volume fractions greater than 1\% [15-17]. Since the NI model does not take the shear dependence of MVE into account, this model is not sufficient qualitatively and quantitatively to explain the viscosity changes in an interacting ferrofluid system. An alternative approach to ferrofluid dynamics and their MVE via the macroscopic thermodynamics was proposed by Müller and Liu [18]. Since our aim here is to better understand the relation between MVE and underlying structural changes, we focus on the kinetic chain model of Zubarev and Iskakova [19]. Since dipolar interactions lead to chain-like aggregates, the chain model assumes the aggregates to be effectively rigid and non-interacting. A few numerical simulations focussed on the anisotropy of magnetoviscous effect in semi-dilute and moderately interacting ferrofluids [20, 21]. The numerical results are successfully compared to the chain model prediction for moderately interacting system. The anisotropy of magnetoviscous effect by varying dipolar interaction strengths including strong interactions is important to study in order to obtain a clear picture of the rheology of various ferrofluid systems. Here, we mainly focus on this aspect, which has not been studied systematically in experiments and in simulations so far. The inter-chain interactions can not be ignored with increasing dipolar interactions. The chain model is not valid in such cases. We provide structural information from simulations to incorporate in the chain model to observe the possible influence of inter-chain interactions.

We organize the paper as follows. In Sec. II, we describe the model we use to study ferrofluids and the nonequilibrium simulation details. In Sec. III, we provide a brief review of existing theoretical models which relate the MVE to mesoscopic structures. Our results and dis- 
cussions on the zero field case are given in Sec. IV and on the applied field case are given in Sec. V. The comparison of our results with experiments is shown in Sec. VI. The summary of the paper and a short discussion are provided in Sec. VII.

\section{METHODS}

We study ferrofluids that can be modelled as a system of $N$ magnetically hard point dipoles in a volume $V$ and diameter $\sigma$ with short range repulsive interaction and long range dipole-dipole interaction [22-24]. We choose truncated and shifted Lennard-Jones potential $U_{i j}^{\mathrm{LJ}}$ with a cut-off $r_{\mathrm{c}}=2^{1 / 6} \sigma$ such that the potential is cut off in the minimum (known as the Weeks-ChandlerAnderson potential [22]), which mimics the steric repulsion of polymer-coated colloidal particles.

$$
U_{i j}^{\mathrm{LJ}}=4 \epsilon\left[\left(\frac{\sigma}{r_{i j}}\right)^{12}-\left(\frac{\sigma}{r_{i j}}\right)^{6}\right],
$$

where $\epsilon$ is the depth of the potential, $r_{i j}$ is the distance between two particles. The long range dipole-dipole interaction is given by

$$
U_{i j}^{\mathrm{dd}}=\frac{\mu_{0} m^{2}}{4 \pi r_{i j}^{3}}\left[\mathbf{u}_{i} \cdot \mathbf{u}_{j}-3\left(\mathbf{u}_{i} \cdot \hat{\mathbf{r}}_{i j}\right)\left(\mathbf{u}_{j} \cdot \hat{\mathbf{r}}_{i j}\right)\right] .
$$

For a system of monodispersed particles, $m$ is the magnetic dipole moment, $d_{\mathrm{m}}$ is the magnetic core diameter, $\mathbf{u}_{i}$ is the orientation of particle $i, \mathbf{r}_{i j}=\mathbf{r}_{i}-\mathbf{r}_{j}$ is the connector vector of the two particles, $r_{i j}=\left|\mathbf{r}_{i j}\right|, \hat{\mathbf{r}}_{i j}=\frac{\mathbf{r}_{i j}}{r_{i j}}$ and $\mu_{0}=4 \pi \times 10^{-7} \mathrm{H} / \mathrm{m}$. The dipole interaction strength of two dipoles with minimum distance $d_{\mathrm{m}}$ is given by $\lambda_{\mathrm{m}}=\mu_{0} m^{2} / 4 \pi k_{\mathrm{B}} T d_{\mathrm{m}}^{3}$, where $k_{\mathrm{B}}$ is the Boltzmann constant and $T$ is the temperature. Due to steric repulsion the minimum distance between two dipolar particles is $d_{\mathrm{h}}$, thus the effective dipolar interaction strength becomes $\lambda=\lambda_{\mathrm{m}}\left(\frac{d_{\mathrm{m}}}{d_{\mathrm{h}}}\right)^{3}$. We take the long-range part of dipolar interaction into account using reaction field method (RF) [25]. In this method, a cavity of radius $r_{\mathrm{RF}}$ (here $r_{\mathrm{RF}}=8.0 \sigma$ ) is defined within which the interactions of dipolar particles are treated explicitly. The particles outside this cavity form a dielectric continuum, $\varepsilon_{\mathrm{s}}$, which develops a reaction field inside the cavity. The strength of the reaction field acting on particle $i$ is given by

$$
\mathbf{H}_{i}^{\mathrm{RF}}=\frac{2\left(\varepsilon_{\mathrm{s}}-1\right)}{2 \varepsilon_{\mathrm{s}}+1} \frac{1}{r_{\mathrm{RF}}^{3}} \sum_{j} \mathbf{m}_{j},
$$

where the summation extends over molecules inside the cavity. We consider $r_{\mathrm{RF}}$ much greater than $r_{\mathrm{c}}$ of short range interactions. We used $\mathrm{RF}$ method with a metallic boundary condition $\left(\varepsilon_{\mathrm{s}} \rightarrow \infty\right)[22,25]$ so that the system is surrounded by a uniform medium with infinite magnetic permeability, thus the internal magnetic field coincides with the applied homogeneous external field $\mathbf{H}$.

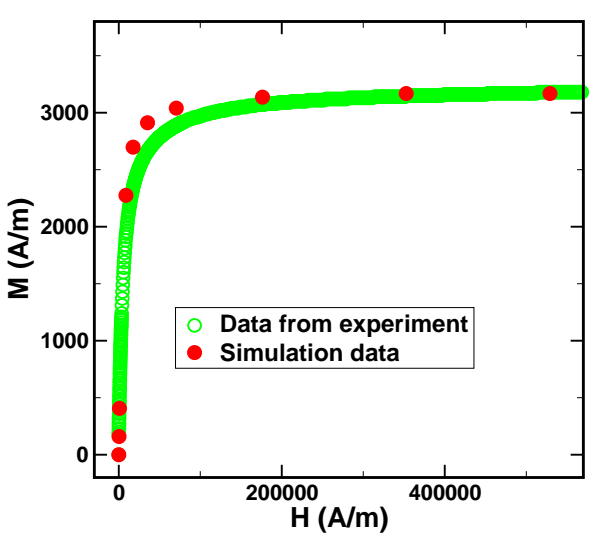

FIG. 1. The equilibrium magnetisation curve $(M)$ as a function of applied magnetic field strength $(H)$.

We have compared the results obtained by RF method with those of the Ewald summation method both with a metallic boundary condition. For the present range of parameters, we find that both methods give identical results within numerical uncertainties. Since we find the RF method to be computationally more efficient than the Ewald summation method, we choose to perform our simulations with the RF method.

We parameterise our model for cobalt-based ferrofluids according to the ferrofluids used in the work by M. GerthNoritzsch et al. [26]. We choose the magnetic volume fraction $\phi=0.0021$ that corresponds to a hydrodynamic volume fraction $\phi_{\mathrm{h}}=\phi\left(d_{\mathrm{h}} / d_{\mathrm{m}}\right)^{3}=0.007$ where magnetic core diameter $d_{\mathrm{m}}=10 \mathrm{~nm}$ and effective hydrodynamic particle diameter $d_{\mathrm{h}}=\sigma=15 \mathrm{~nm}$. The individual magnetic moments $\mathbf{m}_{i}=m \mathbf{u}_{i}$ give rise to a macroscopic magnetization $\mathbf{M}=M_{\mathrm{S}} \overline{\mathbf{u}}$, where $\overline{\mathbf{u}}=(1 / N) \sum_{i} \mathbf{u}_{i}$ is the average orientation of the particles and $M_{\mathrm{s}}=N \mathrm{~m} / \mathrm{V}$ is the saturation magnetization. We choose the saturation magnetization value $M_{\mathrm{s}}=3.2 \times 10^{3} \mathrm{~A} / \mathrm{m}$ from experiment [26]. For a dipolar interaction strength $\lambda \approx 4.6$, the magnetization curve obtained from simulations are comparable to the experimental data as shown in Fig. 1.

We perform Langevin dynamics (LD) simulations in the presence of a shear flow $\mathbf{V}(\mathbf{r})$ with vorticity $\boldsymbol{\Omega}(\mathbf{r})=$ $\frac{1}{2} \boldsymbol{\nabla} \times \mathbf{V}(\mathbf{r})$. The translational and rotational Langevin equations of motion of the system are given by

$$
M \dot{\mathbf{v}}_{i}=-\xi^{\mathrm{T}}\left[\mathbf{v}_{i}(t)-\mathbf{V}(\mathbf{r})\right]+\mathbf{f}_{i}^{\mathrm{B}}(t)+\mathbf{F}_{i}
$$

$\mathbf{I} \cdot \dot{\boldsymbol{\omega}}_{i}=-\xi^{\mathrm{R}}\left[\boldsymbol{\omega}_{i}(t)-\boldsymbol{\Omega}(\mathbf{r})\right]+\boldsymbol{\tau}_{i}^{\mathrm{B}}(t)+\boldsymbol{\tau}_{i}+m \mathbf{u}_{i} \times \mathbf{H}$,

where $M$ and $\mathbf{I}$ are the mass and inertia tensor of the particle with linear and angular velocity $\mathbf{v}_{i}$ and $\boldsymbol{\omega}_{i}$ respectively, $\xi^{\mathrm{T}}$ is the translational friction coefficient and $\xi^{\mathrm{R}}$ is the rotational friction coefficient. For a solvent of viscosity $\eta_{\mathrm{s}}, \xi^{\mathrm{T}}=3 \pi \eta_{\mathrm{s}} \sigma$ and $\xi^{\mathrm{R}}=\pi \eta_{\mathrm{s}} \sigma^{3}$. $\mathbf{f}_{i}^{\mathrm{B}}(t)$ and 
$\boldsymbol{\tau}_{i}^{\mathrm{B}}(t)$ are Gaussian random forces and torques. The potential forces are given by $\mathbf{F}_{i}=-\nabla_{\mathbf{r}_{i}} U$ with $U=$ $\frac{1}{2} \sum_{i j}\left(U_{i j}^{\mathrm{LJ}}+U_{i j}^{\mathrm{dd}}\right)+\sum_{i} U_{i}^{\mathrm{RF}}$, where $U_{i}^{\mathrm{RF}}$ is the potential energy from the reaction field approach. The potential torques are given by $\mathbf{F}_{i}=-\nabla_{\mathbf{r}_{i}} U$ and $\boldsymbol{\tau}_{i}=-\mathcal{L}_{i} U$ with rotational operator $\mathcal{L}_{i}=\mathbf{u}_{i} \times \frac{\partial}{\partial \mathbf{u}_{i}}$. The external magnetic field contributes an additional term $U_{i}^{H}=-m \mathbf{u}_{i} \cdot \mathbf{H}$ to the potential energy of the system. The torque exerted by the magnetic field is $-\mathcal{L}_{i} U_{i}^{H}=m \mathbf{u}_{i} \times \mathbf{H}$. We consider the free-draining limit in Eqs. (3) and (4) since hydrodynamic interactions are not taken into account.

In a planar shear flow along the xy-plane $\mathbf{V}(\mathbf{r})=$ $(\dot{\gamma} y, 0,0)$ with vorticity along the $\mathrm{z}$-axis, the effective shear viscosity is defined as $\eta=-P_{\mathrm{yx}} / \dot{\gamma}$, where $P_{\mathrm{yx}}$ is the yx-component of the pressure tensor and $\dot{\gamma}=\frac{\partial v(x)}{\partial y}$ is the shear rate. In the absence of an external torque, particles rotate with an angular velocity equal to the vorticity $(\boldsymbol{\omega}=\boldsymbol{\Omega})$. A deviation in the angular velocity from the vorticity of the particle causes an additional friction in the rotation of the particle gives rise to a rotational viscosity. In the presence of an external magnetic field, dipole moment of particles prefer to orient in the field direction. A viscous torque $-\xi^{\mathrm{R}}\left(\boldsymbol{\omega}_{i}-\boldsymbol{\Omega}\right)$ tends to rotate the particle causing a misalignment of moment and the field. A magnetic torque $m \mathbf{u}_{i} \times \mathbf{H}$ develops to counteract the viscous torque. From the magnetic and viscous torque balance, we get $\mathbf{M} \times \mathbf{H}=\frac{N \xi^{\mathrm{R}}}{V}(\langle\boldsymbol{\omega}\rangle-\boldsymbol{\Omega})=$ $6 \eta_{\mathrm{s}} \phi_{\mathrm{h}}(\langle\boldsymbol{\omega}\rangle-\boldsymbol{\Omega})[27]$, which contributes to the antisymmetric part of viscous stress tensor. The total viscous stress tensor $\boldsymbol{\sigma}$ can be decomposed into its isotropic, symmetric traceless and antisymmetric part $[4,6]$ :

$$
\boldsymbol{\sigma}=-p \mathbf{I}+\boldsymbol{\sigma}^{\mathrm{sym}}+\frac{1}{2} \boldsymbol{\epsilon} \cdot \boldsymbol{\sigma}^{\mathrm{a}},
$$

where the isotropic pressure $p$ is one-third of the trace of the total viscous pressure tensor. $\boldsymbol{\sigma}^{\mathrm{sym}}$ is the symmetric traceless part of viscous stress tensor. The antisymmetric part of the viscous stress tensor is due to the body coupling $\boldsymbol{\sigma}^{\mathrm{a}}=\mathbf{M} \times \mathbf{H}$. $\boldsymbol{\epsilon}$ is the total antisymmetric tensor of rank three. By incorporating the Irving-Kirkwood expression for the stress tensor, the constitutive equation (the expression for viscous pressure tensor) has a form given by [28]

$$
\begin{array}{r}
\boldsymbol{\sigma}=-p \mathbf{I}+\eta_{\mathrm{s}}\left[\nabla \mathbf{v}+(\nabla \mathbf{v})^{T}\right]-\frac{1}{2 V} \sum_{j<k}^{N}\left(\mathbf{r}_{j k} \mathbf{F}_{j k}\right)^{\mathrm{sym}} \\
+\frac{1}{2} \boldsymbol{\epsilon} \cdot(\mathbf{M} \times \mathbf{H}) .(6)
\end{array}
$$

The rotational viscosity $\left(\eta_{\text {rot }}\right)$ of the system is given by,

$$
\eta_{\text {rot }}=\frac{1}{2 \dot{\gamma}} \boldsymbol{\epsilon} \cdot \boldsymbol{\sigma}^{\mathrm{a}}=\frac{1}{2 \dot{\gamma}} \boldsymbol{\epsilon} \cdot(\mathbf{M} \times \mathbf{H}),
$$

The contribution of $\mathbf{H}_{i}^{\mathrm{RF}}$ to the rotational viscosity is negligible for the range of state points we study and is not included in the calculation of $\eta_{\text {rot }}$. Three Miesowicz viscosity coefficients are defined in a planar shear flow according to the orientation of an external magnetic field with respect to the flow, gradient of the flow and the vorticity direction, denoted as $\eta_{1}, \eta_{2}$, and $\eta_{3}$, respectively $[7,28]$. The relative difference of each viscosity coefficient with respect to the corresponding zero field viscosity determines the anisotropy of magnetoviscous effect $[1,21]$,

$$
\text { MVE, } \Delta \eta=\frac{\eta(H, \dot{\gamma})-\eta(H=0, \dot{\gamma})}{\eta(H=0, \dot{\gamma})} \text {. }
$$

We perform Langevin dynamics simulations for systems containing $N=1000$ particles in a planar shear flow subject to various orientations and strengths of an externally applied magnetic field $\mathbf{H}$. We use Lees-Edwards periodic boundary conditions for non-equilibrium simulations [25]. The integration time step for equilibrium simulations is $\Delta t=0.002$ and reduced to $\Delta t=0.00025$ for high shear rates.

\section{THEORETICAL MODELS}

We briefly review the existing theoretical models developed to understand the rheology of ferrofluids. The magnetoviscous effect of non-interacting ferrofluids has been predicted with the help of the NI model by Shliomis [10]. The rotational viscosity of a non-interacting ferrofluid system predicted using NI model has the form $\eta_{\text {rot }}=M_{\perp} H / 4 \Omega$, where $M_{\perp}$ is the non-equilibrium component of the magnetization perpendicular to the magnetic field direction. For weak flows, $M_{\perp}$ is small and calculated from the phenomenological magnetization equation in linear approximation. The modified rotational viscosity is the predicted as [27],

$$
\eta_{\mathrm{rot}}=\frac{3}{2} \eta_{\mathrm{s}} \phi_{\mathrm{h}} h \frac{L_{1}(h)}{2+h L_{1}(h)}
$$

where $h=m H / k_{\mathrm{B}} T$ is the Langevin parameter and the Langevin function $L_{1}(x)=\operatorname{coth}(x)-1 / x$. According to Shliomis model only rotational viscosity contributes to the total viscosity, $\eta_{1}=\eta_{2}$ and $\eta_{3}=\eta_{\mathrm{s}}$. The maximum viscosity increase predicted by the NI model is $\frac{3}{2} \eta_{\mathrm{s}} \phi_{\mathrm{h}}$. Thus, the relative increase in viscosity is small for low volume fractions. This is not true in the case of interacting particles. Weak inter-particle interactions have been incorporated into dynamic mean-field models [29], but their range of validity is rather limited [20]. Strong dipolar interactions have successfully been included in the chain model [19] with following assumptions that (i) the chains are rigid and straight (ii) dipole moment is frozen in the particle and (iii) inter-chain interactions are neglected. From the minimization of an approximated free energy expression, the chain model predicted the cluster size distribution $g(n)$. The cluster size distribution is predicted to be an exponential function of chain size at strong interaction strengths, $g(n) \propto \exp \left(-n / a_{0}\right)$, where $a_{0}$ is related to the average chain length $\langle n\rangle$ by 
$\langle n\rangle=\left[1-\exp \left(-1 / a_{0}\right)\right]^{-1}$. In the case of a simple shear flow geometry, $\mathbf{v}=\left(v_{x}(y), 0,0\right)$ with flow, gradient and vorticity in $\mathrm{x}, \mathrm{y}$ and $\mathrm{z}$ directions respectively, the shear viscosity predicted by the chain model when the external magnetic field is oriented parallel and perpendicular to the flow direction is given by [19]

$$
\begin{array}{r}
\frac{\eta_{p}-\eta_{\mathrm{s}}}{\eta_{\mathrm{s}}}=\sum_{n} n v g(n)\left(\alpha_{n}+\frac{1}{2}\left[\left(\zeta_{n}+\beta_{n} B\right)\left(\left\langle e_{x}^{2}\right\rangle+\left\langle e_{y}^{2}\right\rangle\right)\right.\right. \\
\left.+(-1)^{p} \beta_{n}\left(\left\langle e_{y}^{2}\right\rangle-\left\langle e_{x}^{2}\right\rangle\right)+2\left(\chi_{n}-2 B \beta_{n}\right)\left\langle e_{x}^{2} e_{y}^{2}\right\rangle\right] \\
\left.+(-1)^{p} \frac{1}{2 v} \frac{\alpha k_{\mathrm{B}} T}{\eta_{\mathrm{s}}}\left(\frac{a_{x}}{2 \dot{\gamma}}\left\langle e_{x}^{2}\right\rangle+\frac{b_{x y}}{\dot{\gamma}}\left\langle e_{x}^{2} e_{y}\right\rangle\right)\right)
\end{array}
$$

where $p=1$ or 2 if the magnetic field is oriented along the flow or the gradient of flow direction, respectively. $a_{x}$ and $b_{x y}$ are calculated from equations for the first and second moments of the orientation distribution function. All parameters are explained in detail in [19]. Since the cluster size distribution $g(n)$ is input to the viscosity expression, Eq. (10), the shear thinning behavior is not properly account in for the model. An ad-hoc solution was proposed in $[1,30]$, where a maximum chain size is associated with each shear rate via a simple force balance. According to this a chain breaks when viscous and magnetic forces become equal. The magnetic interaction force is considered between the particles in the centre of the chain. The viscous force on the chain is estimated as $F_{\text {vis }}=\xi_{\text {eff }} \dot{\gamma} \bar{y}$, where $\xi_{\text {eff }}=\frac{n}{2} \xi^{\mathrm{T}}$ and $\bar{y}=\frac{n}{2} \sigma$, which increases with $\dot{\gamma}$. The magnetic force between particles is estimated by $F_{\mathrm{m}} \sim \lambda k_{\mathrm{B}} T / \sigma$ and does not depend on the chain length. Therefore, a chain disintegrates when $\dot{\gamma}_{\mathrm{c}}=\frac{4 \lambda}{\tau_{\mathrm{B}} n^{2}}$ where $n_{\max }$ is the critical chain length for breakage and $\dot{\gamma}_{c}$ is the critical shear rate.

\section{RESULTS - ZERO APPLIED FIELD}

The ferrofluid system subjected to a planar shear flow exhibits interesting rheological properties both in the absence and presence of an external magnetic field. With the aid of structural properties, we interpret the changes in rheological properties at various shear rates, strengths and orientations of an external magnetic field.

We show in Fig. 2, the zero-field shear viscosity as a function of shear rate for the three chosen dipolar interaction strengths. The shear rates vary by four orders of magnitude $\left(10^{-3} \leq \dot{\gamma} \leq 10\right)$. At low shear rates, the system shows a clear Newtonian regime. In this regime the viscosity is constant with shear rate and shear viscosities for all three $\lambda$ values match with their respective equilibrium viscosities obtained from the GreenKubo (GK) method [24]. With increasing shear rate the system shows a shear-thinning behaviour, where the onset decreases to lower shear rates as dipolar interaction strength increases. We discuss below these properties and associated structural changes for each $\lambda$ value.

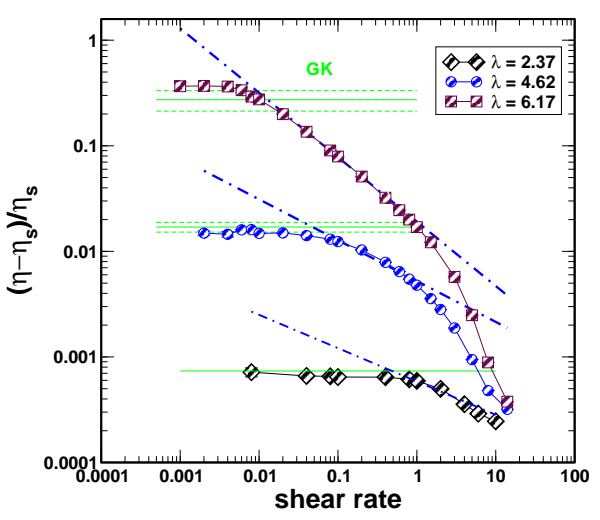

FIG. 2. The zero shear viscosity scaled by solvent viscosity as a function of shear rate for different dipolar interaction strengths $(\lambda)$. A clear transition from Newtonian regime to shear thinning regime with increasing shear rate is observed. The horizontal continuous lines are equilibrium viscosities obtained from the Green-Kubo (GK) method [24] and dashed lines are error bars of equilibrium viscosities.

\section{A. Weak dipolar interaction strength}

In the case of weak dipolar interactions (diamond symbols in Fig. 2), we observe a well defined Newtonian regime for a wide range of shear rates and a feeble shear thinning regime beyond $\dot{\gamma}>1$. The shear thinning regime is fitted with a power law $\frac{\eta-\eta_{\mathrm{s}}}{\eta_{\mathrm{s}}} \propto \dot{\gamma}^{-q}$, where $q$ is the power-law exponent. We identify the onset of shear thinning regime by the intersection of equilibrium viscosity and the power law fit to the shear thinning regime. We want to relate viscosity changes to the structural reorganization of particles from their equilibrium micro-structures. We compute the cluster size distribution $g(n)$, the local connectivity $C\left(n_{\mathrm{c}}\right)$ of our system at different shear rates and compare with the equilibrium case, shown in Fig. 3(a) and Fig. 3(b) . The connectivity computes the fraction of particles having $n_{c}$ number of neighbours. Two particles are considered neighbors if they are separated by a distance less than $1.5 \sigma$, i.e., in the middle between first and second coordination shells [24]. The same criteria is adopted in the cluster size distribution to recognize particles in the same cluster. From the local connectivity analysis (Fig. 3(b)), we observe that for the weak interaction strength $\lambda=2.37$, there is no significant structural changes with shear rate (except for a negligible increment in isolated particles at the cost of one-coordinated particles). The cluster size distribution (Fig. 3(a)) does not vary with shear rate, except for the highest shear rate $\dot{\gamma}=6$, where we find a small decrease in chain length. This small change in the cluster size seems to be contributing to the decrease in the viscosity at high shear rates. In the weak interaction case, we observe no prominent structure formation 


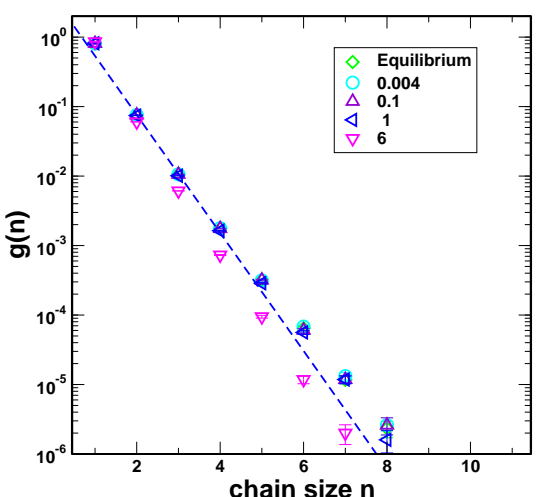

(a)

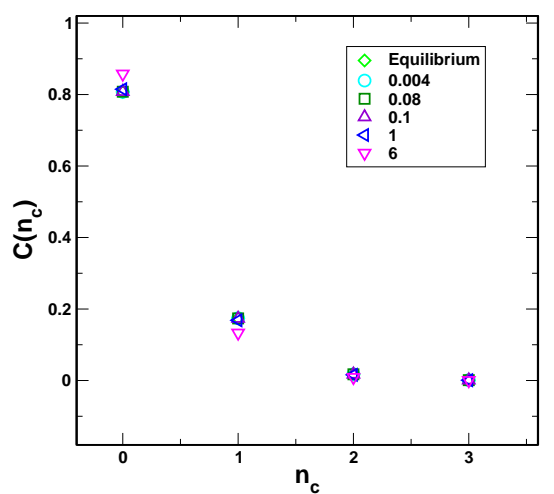

(b)

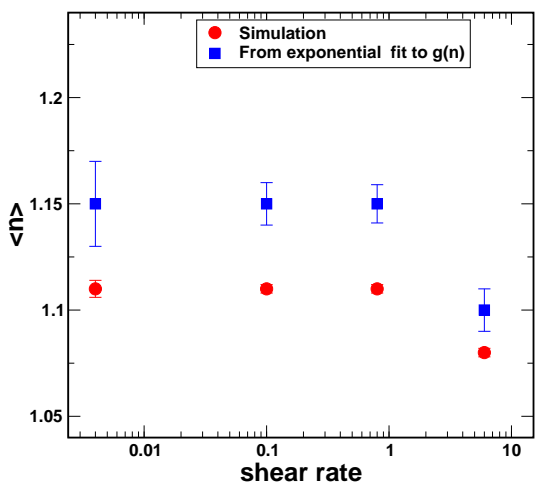

(c)

FIG. 3. For weak dipolar interaction strength $\lambda \approx 2.37$ : (a) the cluster size distribution $g(n)$ as a function of chain size $n$ for various shear rates and the exponential fit is shown as dashed line (b) The connectivity $C\left(n_{\mathrm{c}}\right)$ as a function of coordination number $n_{\mathrm{c}}$ for various shear rates (c) average chain size $\langle n\rangle$ as a function of shear rate. Explanation given in Sec. IV A. in the system, thus the chain model is invalid in this case. Nevertheless, we attempt to fit $g(n)$ obtained from simulations with above mentioned exponential function. We find that $g(n)$ shows an exponential decay but the average chain size $\langle n\rangle$ obtained from the exponential fit shows a deviation from the $\langle n\rangle$ computed from simulation (see Fig. 3(c)). The deviation is expected for weak interaction strengths since the chain model is valid for those systems with straight and rigid chains.

\section{B. Intermediate dipolar interaction strength}

In the case of intermediate dipolar interaction strengths (circles in Fig. 2), the zero field shear viscosities obtained as a function of shear rate have higher values than those observed for weakly interacting ferrofluids due to the micro-structure formation in the system. We observe a prominent shear thinning regime. The onset of shear thinning regime shifts to lower shear rate value compared to that in the weak interaction case. We understand this behaviour in terms of structural properties, the cluster size distribution Fig. 4(a) and the connectivity Fig. 4(b).

For intermediate interaction strength $\lambda \approx 4.62$, with increasing shear rate, the connectivity analysis shows an increment in isolated and one-neighboured particles at the cost of two-neighboured particles (see Fig. 4(b)). This indicates that particles in longer chains tend to form shorter chains and isolated particles with increasing shear rates. The disintegration ("rupture") of long chains in the system is in agreement with earlier simulation results [31]. With further increase in shear rate (beyond $\dot{\gamma}>0.4$ ), we observe a prominent reduction in particles with one- and two- neighbours and an increase in isolated particles. The cluster size distribution shows a prominent deviation from its equilibrium structure with increasing shear rate. The rupturing is more prominent in this case compared to the weakly interacting ferrofluids (see Fig. 4(a)). The average chain size decays in a stretched exponential form, $\langle n\rangle \propto \exp -(c \dot{\gamma})^{\beta}, \beta<1$, with the shear rate (see Fig. 4(c)). From this analysis we infer that a decrease in one- and two- neighboured particles and shortening of chain size corresponds to a prominent decrease in viscosity with increasing shear rate.

\section{Strong dipolar interaction strength}

In the case of strong dipolar interaction strengths (squares in Fig. 2), we observe a Newtonian regime at shear rates, $\dot{\gamma}<0.01$ and the onset of shear thinning is shifted even more towards low shear rates compared to weak and intermediate dipolar interaction strengths. The shear thinning is prominent in this case. Interestingly, one can distinguish two different shear thinning regimes $(0.01<\dot{\gamma}<2$ and $2<\dot{\gamma})$, characterized by different slopes. The structural analyses are shown in Fig. 5. In 


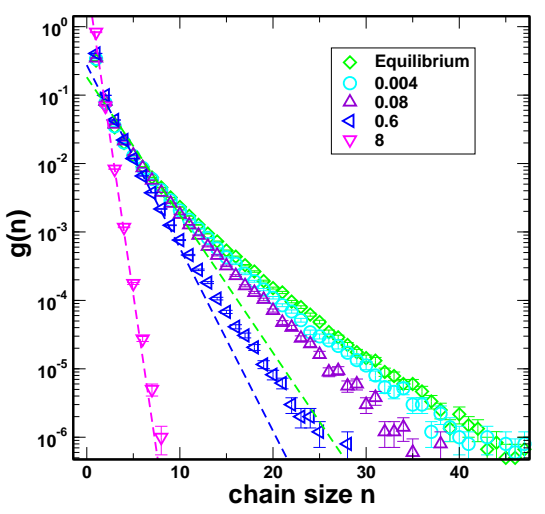

(a)

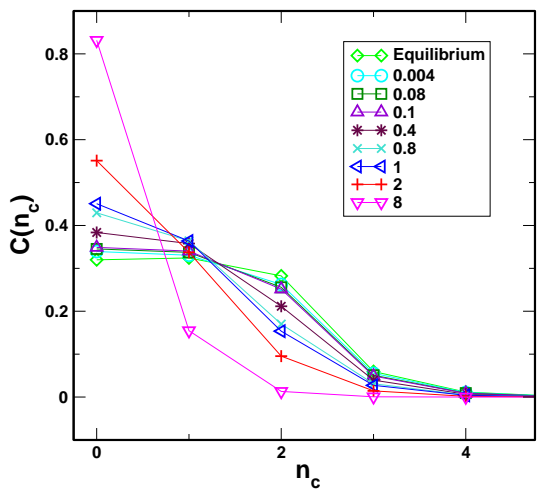

(b)

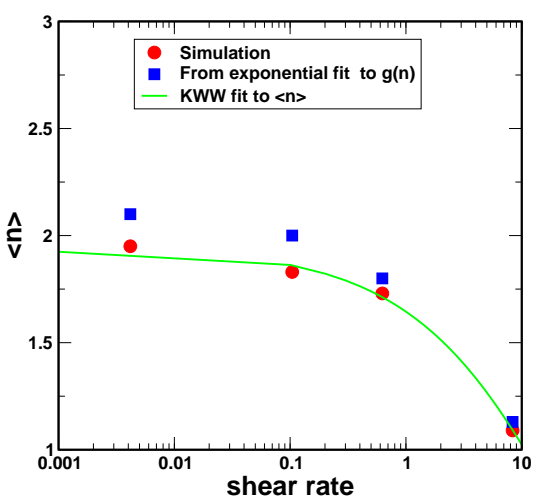

(c)

FIG. 4. For intermediate dipolar interaction strength $\lambda \approx$ 4.62: (a) the cluster size distribution $g(n)$ as a function of chain size $n$ for various shear rates and the exponential fit to the data is shown as dashed line (b) The connectivity $C\left(n_{\mathrm{c}}\right)$ as a function of coordination number $n_{\mathrm{c}}$ for various shear rates (c) average chain size $\langle n\rangle$ as a function of shear rate. Explanation given in Sec. IV B.

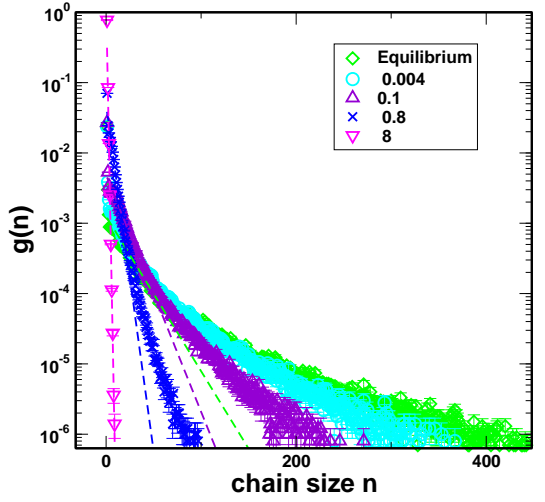

(a)

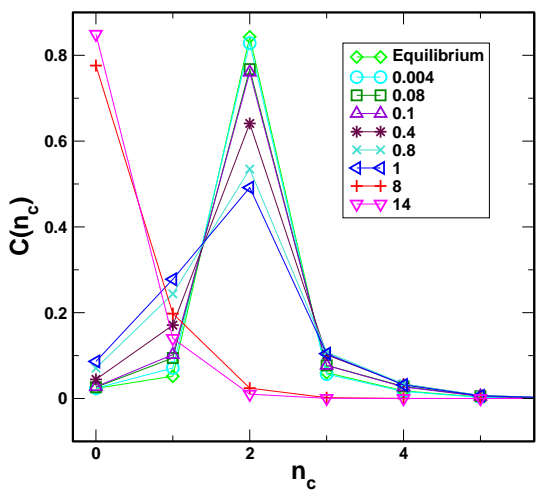

(b)

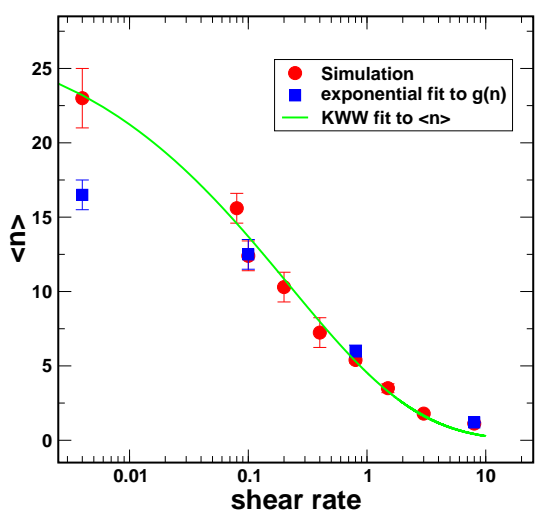

(c)

FIG. 5. For strong dipolar interaction strength $\lambda \approx 6.17$ : (a) the cluster size distribution $g(n)$ as a function of chain size $n$ for various shear rates(b) The connectivity $C\left(n_{\mathrm{c}}\right)$ as a function of coordination number $n_{\mathrm{c}}$ for various shear rates (c) average chain size $\langle n\rangle$ as a function of shear rate. Explanation given in Sec. IV C. 
Fig. 5(a) and Fig. 5(b), we show the cluster size distribution and the connectivity, respectively, for different shear rates. In the Newtonian regime, the connectivity and cluster size distribution do not deviate significantly from the equilibrium case. The connectivity shows that the number of particles having two neighbours are prominent and the cluster size distribution shows the presence of large clusters as in equilibrium. These micro-structures contribute to the high value of viscosity in the Newtonian regime. In the shear thinning regime $0.01<\dot{\gamma} \leq 2$, we observe from the connectivity analysis a gradual increase in the one-neighboured particles at the cost of two-neighboured particles. It is clear from the cluster size analysis that large clusters rupture into smaller ones with increasing shear rate. At higher shear rate values, $\dot{\gamma}>2$, the micro-structures disintegrate mostly into isolated $(80 \%)$ and one-neighboured particles. The cluster size distribution in these regime is in agreement with rupturing observed in connectivity analysis.

The average chain size from simulations is comparable to that obtained from the exponential fit to the cluster size distribution as discussed in the chain model prediction. At the lowest shear rate this simple exponential function fails due to the presence of complex structures. The average chain size decays with shear rate in a stretched exponential form with a stretching exponent $\beta<1$ (see Fig. 5(c)).

Here, we summarise our results pertaining to the zerofield shearing. We have studied the shear viscosity at different shear rates for varying dipolar interaction strengths. In the weak dipolar interaction, we have observed a prominent Newtonian regime for a wide range of shear rates, but with increasing dipolar strength, the system shows a strong shear thinning behaviour. We have studied the structural changes as the system goes from Newtonian to shear thinning regime. We have found that the rupturing of structures play an important role in the decrease in viscosity at high shear rates. Snapshots of ferrofluid system at weak and strong dipolar interaction strengths in Newtonian and shear thinning regime are shown in Fig. 6. Next we focus on rheology of ferrofluids in the presence of an external magnetic field.

\section{RESULTS - APPLIED MAGNETIC FIELD}

The external magnetic field changes the total viscosity of the system with varying field strength and orientation. We study the effect of an applied field in the Newtonian and shear thinning regime. The Miesowicz viscosity coefficients $\eta_{1}, \eta_{2}$ and $\eta_{3}$ are defined corresponding to the orientation of an applied field in the flow, gradient of the flow and the vorticity direction, respectively. Each viscosity coefficient has contributions from the symmetric part (termed as configurational viscosity $\eta_{\text {conf }}$ ) and from the anti-symmetric part of the stress tensor (termed as the rotational viscosity $\eta_{\text {rot }}$ ), both of which we compute explicitly and the total viscosity is $\eta_{\text {total }}=\eta_{\text {conf }}+\eta_{\text {rot }}$

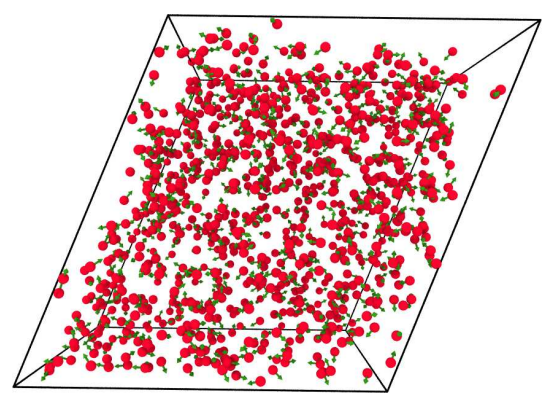

(a) $\lambda=2.37$, Newtonian regime

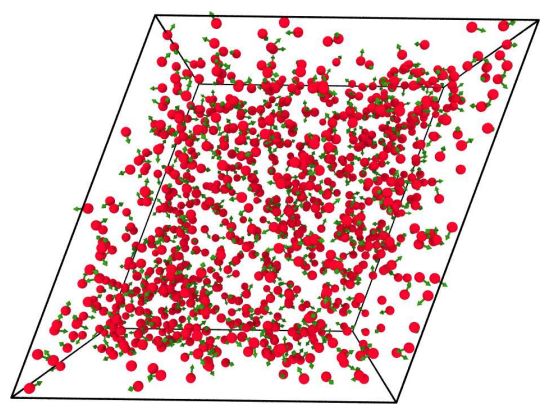

(b) $\lambda=2.37$, Shear thinning regime

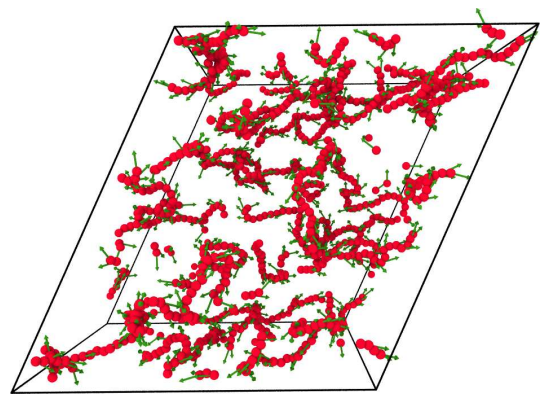

(c) $\lambda=6.17$, Newtonian regime

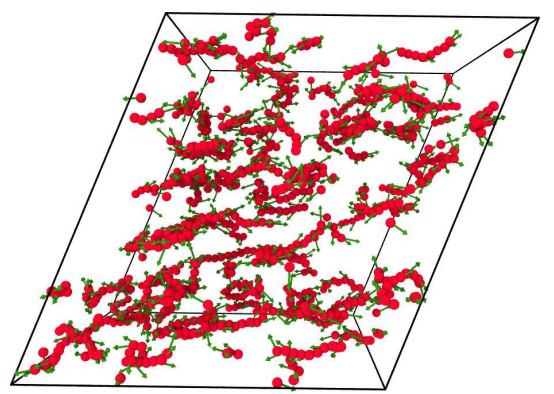

(d) $\lambda=6.17$, Shear thinning regime

FIG. 6. Snapshots (projection of all particles) of model ferrofluid system in a shear flow in the absence of magnetic field $(H=0)$.

(see Eqs. (6) and (7)).

We organize our results in the same format as in the Sec. IV. For the chosen values of dipolar interaction strengths $(\lambda \approx 2.37,4.62$ and 6.17$)$, we have applied an external magnetic field for varying strength, $0.5<|\mathbf{H}|<4$. We first present the results related to the effect of applied field on the shear viscosities in the zero field Newtonian regime. 


\section{A. Applied magnetic field- (i) Newtonian regime}

The Miesowicz viscosities in the Newtonian regime computed at each dipolar interaction strength are shown in Fig. 7 (a) for $(\lambda=2.37, \dot{\gamma}=0.04)$, Fig. $8(\mathrm{a})$ for $(\lambda=$ 4.62, $\dot{\gamma}=0.004)$ and Fig. 9 (a) for $(\lambda=6.17, \dot{\gamma}=0.008)$. The corresponding structural changes are studied for an applied field strength $H_{x}=H_{y}=H_{z}=2$ which corresponds to a Langevin parameter $h \approx 3,4.3,6$ for $\lambda \approx 2.37,4.62$ and 6.17 , respectively.

\section{Weak dipolar interaction strength, $\lambda \approx 2.37$}

At $\lambda=2.37$, the viscosity changes are rather well described by Shliomis prediction, Eq. (9), assuming particles are non-interacting (see dashed lines in Fig. 7(a)). The viscosity is dominated by the rotational contribution leading to $\eta_{1} \approx \eta_{2}$ and $\eta_{3} \approx \eta_{\mathrm{s}}$. Deviations from Shliomis prediction are due to weak inter-particle interactions leading to micro-structure formation. The viscosity change from the zero-field viscosity (MVE), when the field is oriented (1) along flow direction, shows ten times increase in viscosity (contribution from $\eta_{\text {rot }}$ ) (2) along the gradient direction of flow, shows ten times increase in viscosity (contribution from $\eta_{\text {rot }}$ ) (3) along the vorticity direction, shows no change in viscosity (contribution is only from $\eta_{\text {conf }}$ ), see Fig. 10.

Both the structural quantities (the connectivity and the chain size distribution) show same behaviour in all three directions (see Figs. 7(b) and 7(c)), which explains why the configurational viscosity does not change with the direction. Also the value of configurational viscosity is small due to the absence of prominent structure formation (presence of short chains only) in the system.

The rigidity of the chains is extracted from the radius of gyration as $\left\langle R_{\mathrm{g}}^{2}\right\rangle \propto n^{2 \nu}$, where $n$ is the chain size, $\nu$ is the rigidity exponent and it varies as $1 / 2 \leq \nu \leq 1$. The chains are rigid when $\nu$ approaches 1 . A fully flexible chain is described by $\nu=1 / 2$. In the case of $\lambda \approx 2.37$, we find that the rigidity exponent is $\nu \approx 0.7$. Thus we infer that the chains present in the system are semi-flexible hence the misalignment of magnetic moment and the field causes the rotational viscosity which contributes to the magnetoviscous effect. Note that a slightly larger value of $\nu$ was observed in strong magnetic fields for a more concentrated system [31].

\section{Intermediate dipolar interaction, $\lambda \approx 4.62$}

For the case of intermediate dipolar interactions (Fig. 8(a)), we observe that the total viscosity has contributions from both configurational and rotational viscosities. When the applied field is oriented in the flow and gradient direction, $\eta_{\text {conf }}$ and $\eta_{\text {rot }}$ have almost equal contributions. When the field is in the vorticity direction, the sole contribution to the total viscosity is from the

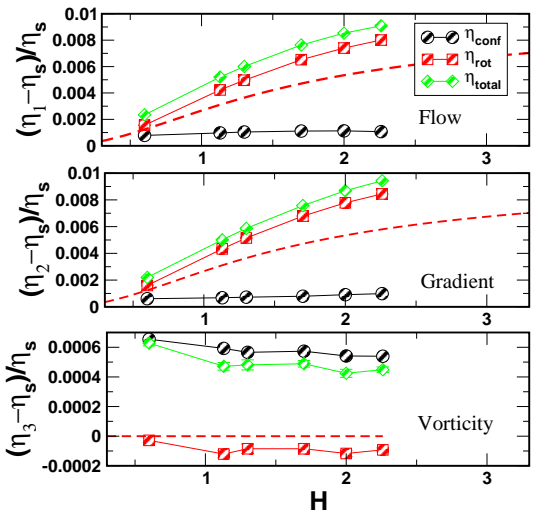

(a)

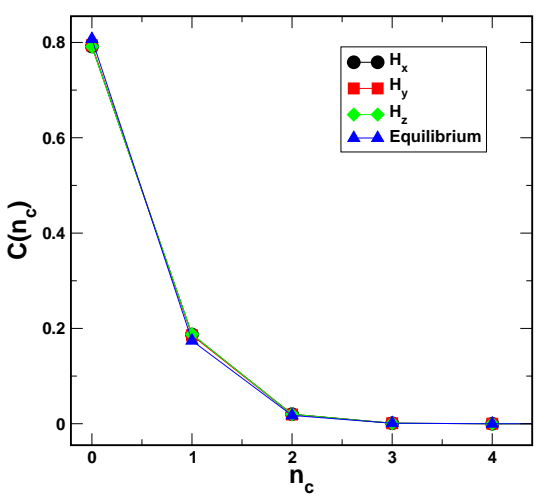

(b)

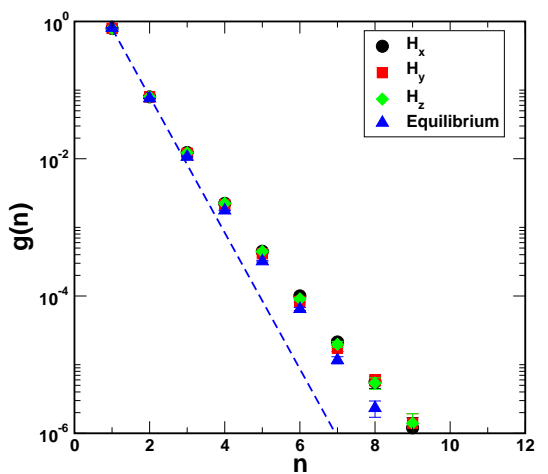

(c)

FIG. 7. For $\lambda \approx 2.37$ with applied field $H$ in Newtonian regime: (a) The configurational viscosity $\eta_{\text {conf }}$, the rotational viscosity $\eta_{\text {rot }}$ and the total viscosity $\eta_{\text {tot }}=\eta_{\text {conf }}+\eta_{\text {rot }}$ as a function of strength of the magnetic field $H$ are shown as circles, squares and diamond symbols, respectively. Top panel: Viscosities when the field is oriented in the flow direction, middle panel: when the field is oriented in the gradient of the flow direction, bottom panel: when the field is oriented in the vorticity direction. (b) The connectivity $C\left(n_{\mathrm{c}}\right)$ as a function of $n_{\mathrm{c}}$ for different orientations of magnetic field (c) Cluster size distribution $g(n)$ as a function of $n$ at different orientations of magnetic field. Exponential fit to $g(n)$ is shown as dashed lines. All structural analysis are performed with a fixed magnetic field strength of $H=2.0$. Explanation is given in Sec. V A 1. 
configurational viscosity. The increase in configurational viscosity with applied magnetic field strength when the field is oriented in the gradient direction can be explained in terms of micro-structural changes. In this case, the connectivity (see Fig. 8(b)) shows a huge deviation from equilibrium structures. The fraction of two-neighboured particles are increased at the cost of isolated particles. The cluster size distribution (see Fig. 8(c)) shows an increase in the number of large clusters. Both quantities show the presence of long chains in the system aligned along the field direction. Due to the prominent chain formation we observe an increase in configurational viscosity. The rotational viscosity increases with the field, when the field is applied in the flow and gradient direction and is zero in the vorticity direction. Qualitatively, we find that this behaviour of $\eta_{1}$ and $\eta_{2}$ is also well predicted by the chain model for chains with an axis ratio of $2-3$ [19]. The viscosity change from the zero-field viscosity (MVE), when the field is oriented (1) along the flow direction, shows four times increase in viscosity (contribution from both $\eta_{\text {conf }}$ and $\eta_{\text {rot }}$ ) (2) along gradient direction of the flow, shows forty times increase in viscosity (contribution from both $\eta_{\text {conf }}$ and $\eta_{\text {rot }}$ ) (3) along the vorticity direction, shows no change in viscosity (contribution is only from $\eta_{\text {conf }}$ ), see Fig. 10 . We find that chains are relatively rigid ( rigidity exponent $\nu \sim 0.8$ ) compared to those present in weakly interacting systems. The misalignment of rigid chains from the magnetic field direction causes an increase in rotational viscosity. Due to the long chain formations in the system, the configurational viscosity also contributes to MVE in the gradient of flow direction. Thus, the contributions from both rotational and configurational viscosities cause the huge increase in MVE.

\section{Strong dipolar interaction, $\lambda \approx 6.17$}

In the case of strong dipolar interactions (Fig. 9(a)), the main contribution to the total viscosity is from the configurational part when the field is oriented along the flow and the vorticity direction. A similar behaviour of the configurational viscosity is qualitatively predicted by the chain model for chain lengths greater than 5 $[6,19]$. The connectivity analysis shows a deviation from the equilibrium structure with an increase in twoneighboured particles at the cost of decreasing threeneighboured particles in the flow and vorticity direction. This explains a decrease in viscosity from the zero field viscosity value. Along the gradient direction the connectivity shows an increase in the one-neighboured particles and a decrease in the three-neighboured particles. This observation is not enough to interpret the increase in configurational viscosity along the gradient direction. We computed cluster size distribution and observed that in the gradient direction the number of chains between lengths $10-40$ has increased considerably at the cost of isolated particles, which explains the increase in con-

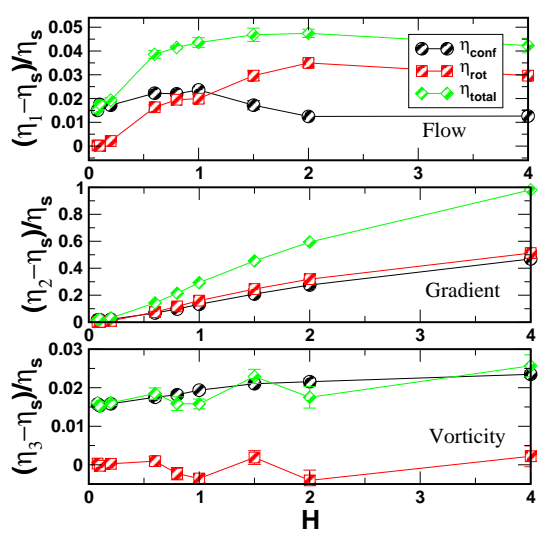

(a)

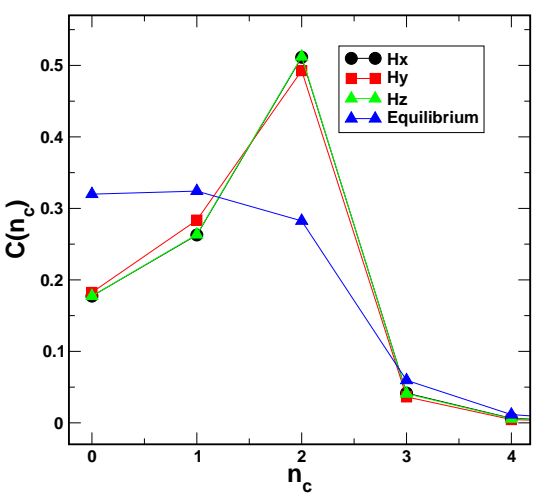

(b)

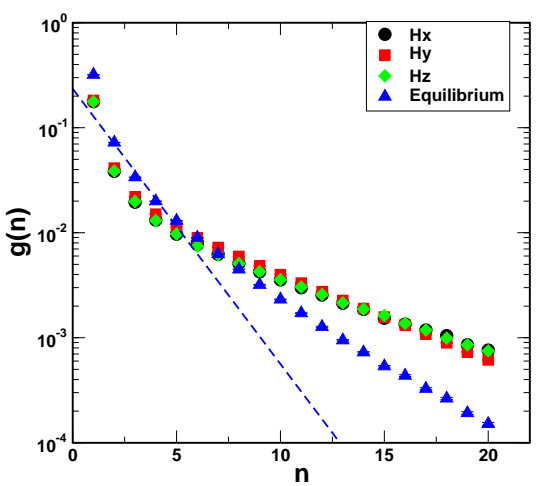

(c)

FIG. 8. For $\lambda \approx 4.62$ with applied field $H$ in Newtonian regime: (a) The configurational viscosity $\eta_{\text {conf }}$, the rotational viscosity $\eta_{\text {rot }}$ and the total viscosity $\eta_{\text {tot }}=\eta_{\text {conf }}+\eta_{\text {rot }}$ as a function of strength of the magnetic field $H$ are shown as circles, squares and diamond symbols, respectively. Top panel: Viscosities when the field is oriented in the flow direction, middle panel: when the field is oriented in the gradient of the flow direction, bottom panel: when the field is oriented in the vorticity direction. (b) The connectivity $C\left(n_{\mathrm{c}}\right)$ as a function of $n_{\mathrm{c}}$ for different orientations of magnetic field (c) Cluster size distribution $g(n)$ as a function of $n$ at different orientations of magnetic field. Exponential fit to the data is shown as dashed lines. All structural analysis are performed with a fixed magnetic field strength of $H=2.0$. Explanation is given in Sec. V A 2. 
figurational viscosity. The rotational viscosity show a negligible change with field oriented along the flow direction and an increase along the gradient of flow direction. At the vorticity direction the rotational viscosity value is zero. The viscosity change from the zero-field viscosity (MVE), when the field is oriented (1) along the flow direction, shows decrease in viscosity (contribution from $\eta_{\text {conf }}$ ) (2) along gradient direction of the flow, shows ten times increase in viscosity (contribution from $\eta_{\text {conf }}$ and $\left.\eta_{\text {rot }}\right)$ (3) along the vorticity direction, shows a decrease in viscosity (contribution is only from $\eta_{\text {conf }}$ ), see Fig. 10. In the flow and the vorticity direction, viscosity decreases from the zero field value due to the absence of complex structures which are present in the zero field case. Also, the rigidity exponent calculated for $\lambda \approx 6.17$ is $\nu \sim 0.9$. Therefore, the chains are very rigid and are more or less perfectly oriented in the field direction. So in the flow and vorticity direction there is no contribution from change in rotational viscosity. In the gradient direction ten times increment in viscosity is due to the misalignment of chains from the field direction and due to the chain formation. Since the zero field viscosity has a huge value due to the complex structure, the change in viscosity is not as prominent as in the case of intermediate interaction strength. The magnetoviscous effect in Newtonian regime is summarised in the schematic shown in Fig. 10.

\section{B. Applied magnetic field- (ii) shear thinning regime}

We study the effect of an external magnetic field in the shear thinning regime recognized in the zero field case as shown in Fig. 2. The chosen shear rate, corresponding to the shear thinning regime, vary with the dipolar interaction strength. For $\lambda \approx 2.37,4.62$ and 6.17 we choose shear rates $\dot{\gamma}=2.0, \dot{\gamma}=0.8$ and $\dot{\gamma}=0.4$, respectively. We begin with the weak dipolar interaction case.

\section{Weak dipolar interaction strength, $\lambda \approx 2.37$}

In the case of weak dipolar interaction, the viscosity changes are well described using Shliomis model, as shown in Fig. 11(a) with red dashed lines. With increasing shear rate, the existing structure ruptures and the smaller fragments act like non-interacting system in a dilute regime. These features are consistent with the structural analysis where we see an increment in the population of isolated particles.

The change in viscosity from the zero-field viscosity, when the field is oriented (1) along the flow direction, shows ten times increases in viscosity (2) along gradient direction of the flow, shows ten times increase in viscosity (3) along the vorticity direction, is negligible, see Fig. 17. We also find that the rigidity exponent (which is around 0.7 ) shows a negligible change with the shear rate

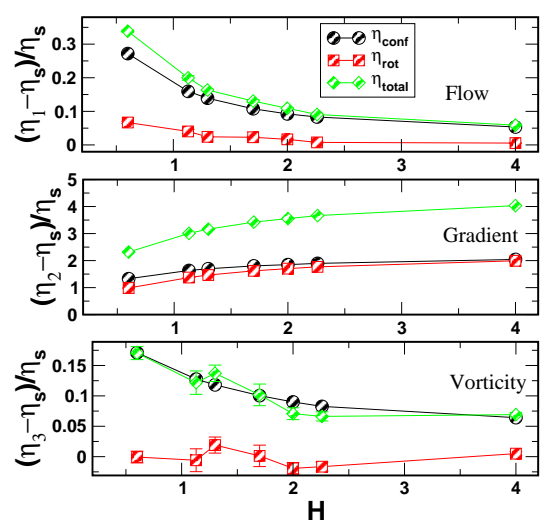

(a)

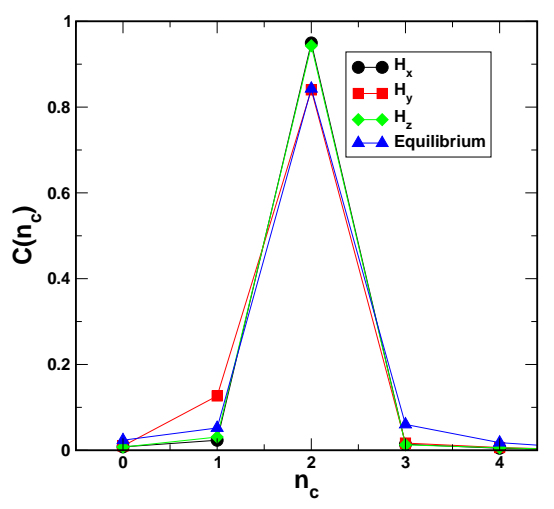

(b)

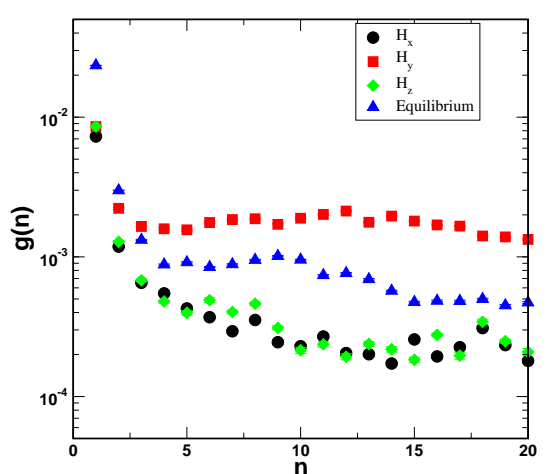

(c)

FIG. 9. For $\lambda \approx 6.17$ with applied field $H$ in Newtonian regime: (a) The configurational viscosity $\eta_{\text {conf }}$, the rotational viscosity $\eta_{\text {rot }}$ and the total viscosity $\eta_{\text {tot }}=\eta_{\text {conf }}+\eta_{\text {rot }}$ as a function of strength of the magnetic field $H$ are shown as circles, squares and diamond symbols, respectively. Top panel: Viscosities when the field is oriented in the flow direction, middle panel: when the field is oriented in the gradient of the flow direction, bottom panel: when the field is oriented in the vorticity direction. (b) The connectivity $C\left(n_{\mathrm{c}}\right)$ as a function of $n_{\mathrm{c}}$ for different orientations of magnetic field (c) Cluster size distribution $g(n)$ as a function of $n$ at different orientations of magnetic field. Exponential fit to the data is given as dashed line. All structural analysis are performed with a fixed magnetic field strength of $H=2.0$. Explanation is given in Sec. V A 3. 


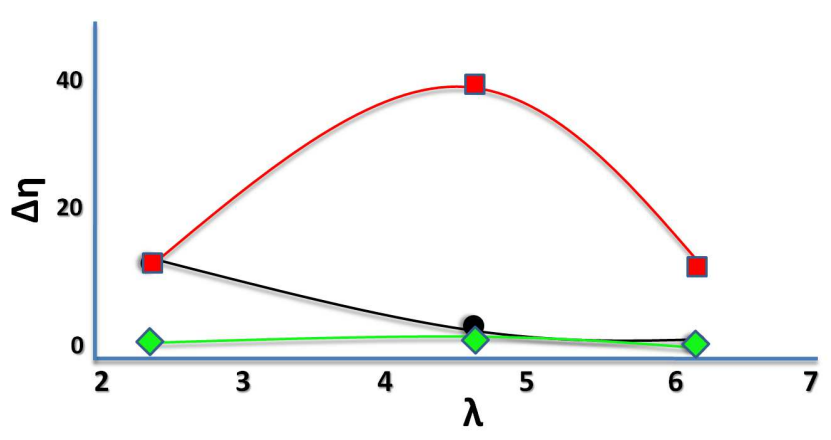

FIG. 10. A schematic of MVE in Newtonian regime for different dipolar interaction strengths. The MVE when the field is oriented in (1) the flow direction is shown as circles (2) the gradient direction of flow is shown as squares (3) vorticity direction is denoted by diamonds.

(see Fig. 12(b)). Thus the only contribution to the magnetoviscous effect is from the rotational viscosity. The change of the configurational viscosity is negligible due to the lack of prominent structural changes.

We have also looked at the shear rate dependency on the viscosity at a fixed applied magnetic field strength $(H=2.0)$, which is shown in Fig. 12(a). We find that at this interaction strength and fixed magnetic field, the viscosity is fairly constant of shear rate. We notice that the chain model is invalid in this case due to lack of chains in the system. In the chain model calculation we have used the $g(n)$ values from the simulation.

\section{Intermediate dipolar interaction strength, $\lambda \approx 4.62$}

In the case of intermediate dipolar interactions (Fig. 13(a)), the total viscosity has contributions from both configurational and rotational viscosity. But the absolute value of viscosities are drastically reduced compared to the Newtonian regime. The structural quantities show that the fraction of isolated particles increases from $20 \%$ (in the Newtonian regime) to $50 \%$ and the fraction of particles having two-neighbours decreases to $10 \%$ in the gradient direction from $50 \%$. This shows clearly that rupturing of the structures is prominent in the gradient direction (see Fig. 13(b)). The cluster size distribution also confirms this observation (Fig.13(c)). The rigidity exponent shows a decrease in it value with shear rate from $\nu \sim 0.85$ to $\nu \sim 0.65$. The rigidity of the chains are affected by the shear rate (Fig. 14(b)). The MVE is less prominent in this case compared to the weakly interacting regime due to the strong rupturing of structures, see Fig. 17.

Now we discuss the shear rate dependence of the viscosity at a fixed magnetic field strength $(H=2.0)$, which is shown in Fig.14(a). We find that at this interaction

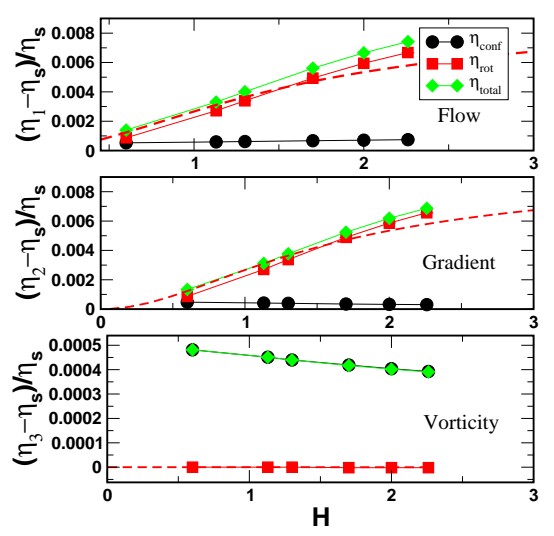

(a)

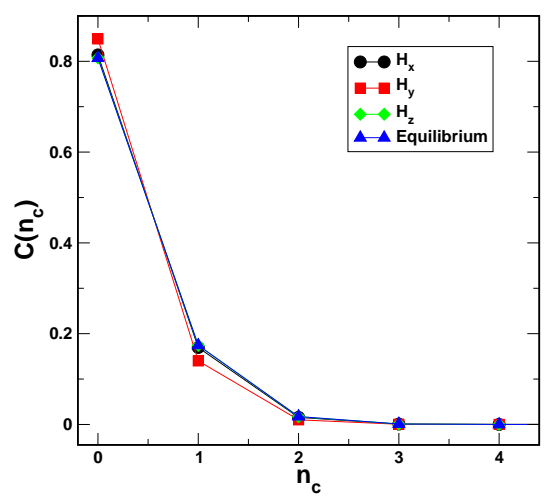

(b)

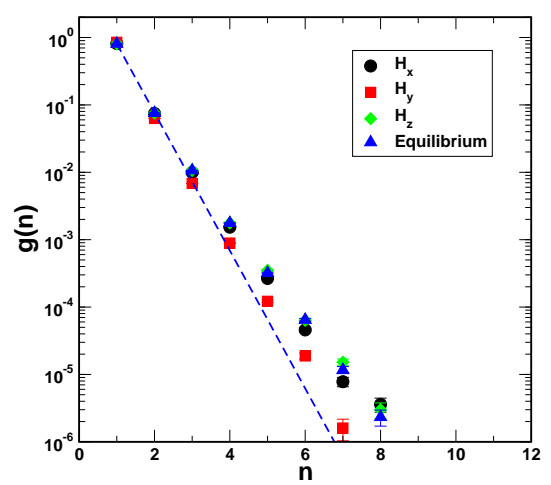

(c)

FIG. 11. For $\lambda \approx 2.37$ with applied field $H$ in shear thinning regime: (a) The configurational viscosity $\eta_{\text {conf }}$, the rotational viscosity $\eta_{\text {rot }}$ and the total viscosity $\eta_{\text {tot }}=\eta_{\text {conf }}+\eta_{\text {rot }}$ as a function of strength of the magnetic field $H$ are shown as circles, squares and diamond symbols, respectively. Top panel: Viscosities when the field is oriented in the flow direction, middle panel: when the field is oriented in the gradient of the flow direction, bottom panel: when the field is oriented in the vorticity direction. (b) The connectivity $C\left(n_{\mathrm{c}}\right)$ as a function of $n_{\mathrm{c}}$ for different orientations of magnetic field (c) Cluster size distribution $g(n)$ as a function of $n$ at different orientations of magnetic field. Exponential fit to the data is given as dashed line. All structural analysis are performed with a fixed magnetic field strength of $H=2.0$. Explanation is given in Sec. VB 1. 


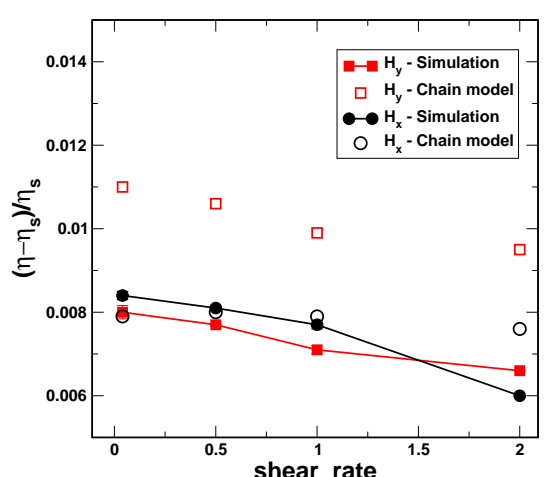

(a)
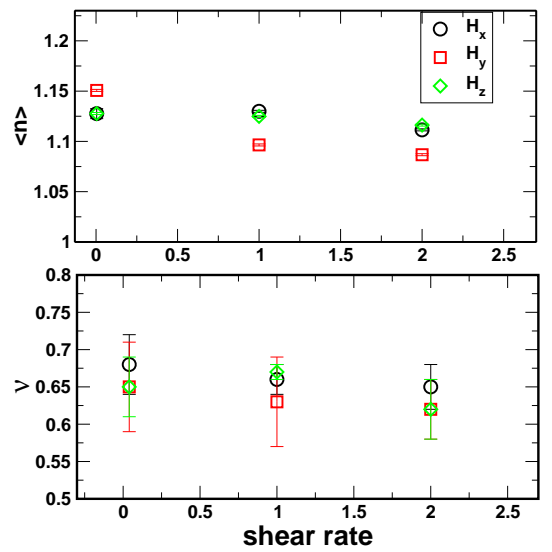

(b)

FIG. 12. For $\lambda \approx 2.37$ : (a) The total viscosities in the flow (circles) and gradient direction of flow (squares) from simulation (solid symbols) and the chain model prediction (open symbols computed by inputting value for chain size distribution from simulations) as a function of shear rate. (b)Top panel: Average cluster size as a function of shear rate. Bottom panel: The rigidity exponent $\nu$ is shown for different orientation of magnetic field as a function of shear rate for the magnetic field strength $H=2.0$.

strength and fixed magnetic field strength we observe a prominent decrease in viscosity with the shear rate in the gradient direction of the flow and there is no significant viscosity change observed in the flow direction. We compare it with the chain model prediction (Fig.14(a)) by inputting the $g(n)$ from simulations. We observe that the average chain size $\langle n\rangle$ decays in a stretched exponential manner with shear rate.

\section{Strong dipolar interaction strength, $\lambda \approx 6.17$}

In the case of strong dipolar interactions (Fig. 15(a)), the total viscosity has a non-monotonic behaviour with increasing magnetic field strength when the field is oriented in the flow direction. The total viscosity increases

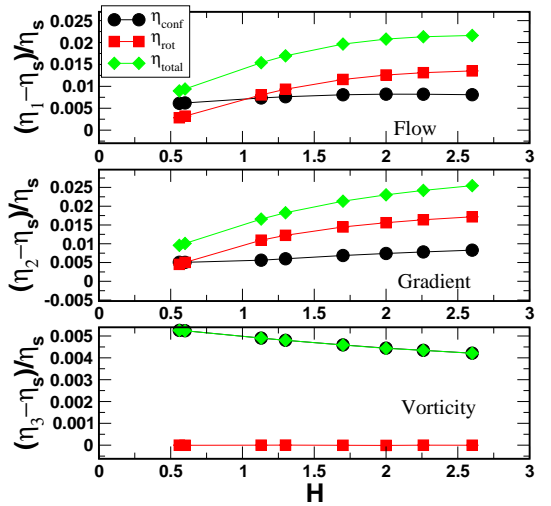

(a)

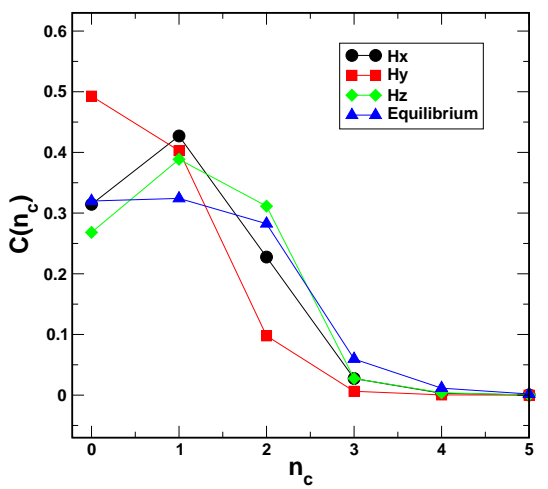

(b)

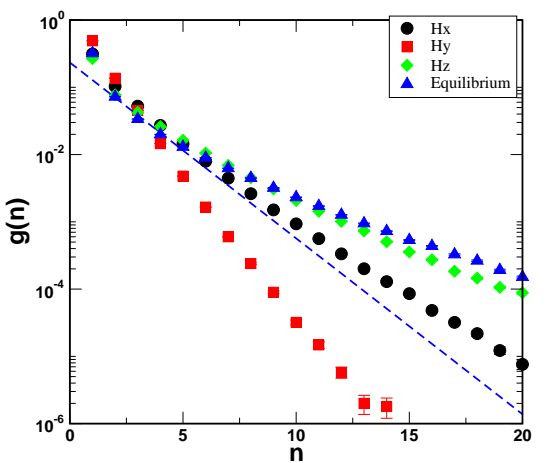

(c)

FIG. 13. For $\lambda \approx 4.62$ with applied field $H$ in shear thinning regime: (a) The configurational viscosity $\eta_{\text {conf }}$, the rotational viscosity $\eta_{\text {rot }}$ and the total viscosity $\eta_{\text {tot }}=\eta_{\text {conf }}+\eta_{\text {rot }}$ as a function of strength of the magnetic field $H$ are shown as circles, squares and diamond symbols, respectively. Top panel: Viscosities when the field is oriented in the flow direction, middle panel: when the field is oriented in the gradient of the flow direction, bottom panel: when the field is oriented in the vorticity direction. (b) The connectivity $C\left(n_{\mathrm{c}}\right)$ as a function of $n_{\mathrm{c}}$ for different orientations of magnetic field (c) Cluster size distribution $g(n)$ as a function of $n$ at different orientations of magnetic field. Exponential fit to the data is denoted by dashed line. All structural analysis are performed with a fixed magnetic field strength of $H=2.0$. Explanation is given in Sec. VB 2 . 


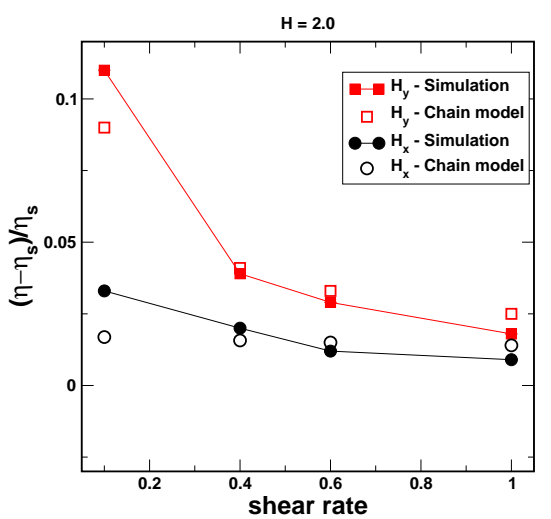

(a)
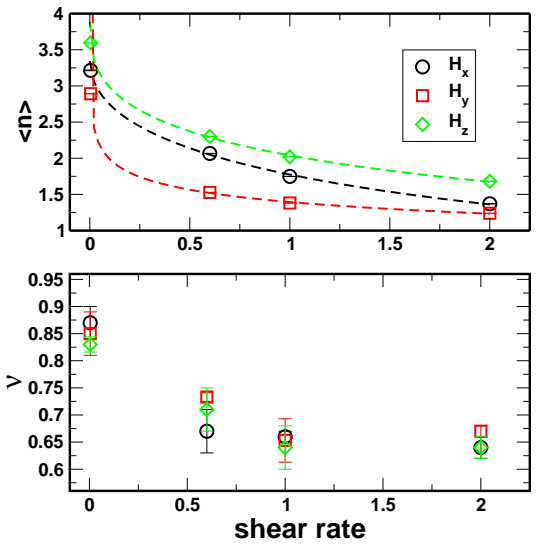

(b)

FIG. 14. For $\lambda \approx 4.62$ : (a) The total viscosities in the flow (circles) and gradient direction of the flow (squares) from simulations (solid symbols) and the chain model prediction (open symbols computed by inputting the values of the chain size distribution from simulations) as a function of shear rate. (b) Top panel: Average cluster size as a function of shear rate. The stretched exponential fit is shown as dashed lines. Bottom panel: The rigidity exponent $\nu$ is shown for different orientation of magnetic field as a function of shear rate for the magnetic field strength $H=2.0$.

with field strength when the field is oriented in the gradient direction and decreases when the field is oriented in the vorticity direction. The configurational viscosity decreases with $H$ in the flow and vorticity direction and increases with $H$ in the gradient direction. The explanation to this behaviour is similar to that given for the viscosity change in strong dipolar interaction strength in the Newtonian regime. The rotational viscosity increase with $H$ both in the flow and gradient direction of the flow and it is zero in the vorticity direction (Fig. 15(a)). The structural quantities show a drastic rupturing of microstructures in gradient direction of the flow and we observe increase in number of chains with short chain length and a decrease in number of chains with large chain length. The rigidity component of chains in the flow and gra- dient direction shows a change in values from $\nu \sim 0.95$ to $\nu \sim 0.65$. In the vorticity direction it ranges form $\nu \sim 0.95$ to $\nu \sim 0.8$. The MVE is similar to that of intermediate interaction strength due to the strong rupturing in the system, see Fig. 17.

Fixing the applied magnetic field strength, we show the change in viscosities as a function of shear rate in Fig. 16(a). Viscosities in flow and gradient direction show a prominent decrease with shear rate. The chain model fails to predict the viscosity at low shear rates in the flow direction.

To summarise this section, we have computed Miesowicz viscosities in the shear thinning regime. We have studied the corresponding structural changes and explained the viscosity changes in terms of rupturing of chains. Rupturing is significant in the intermediate and strong dipolar interaction regime, leading to pronounced shear thinning behavior. Even though the field-induced viscosity is larger for stronger dipolar interactions (compare Figs. 12(a), 14(a), 16(a)), the relative viscosity increase measured by $\Delta \eta$ is the largest in the weakly interacting regime due to the low value of the zero-field viscosity. The magnetoviscous effect in the shear thinning regime is summarised in the schematic shown in Fig. 17. 


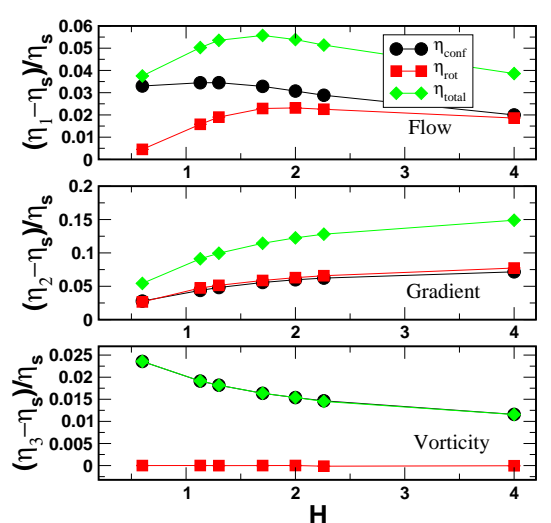

(a)

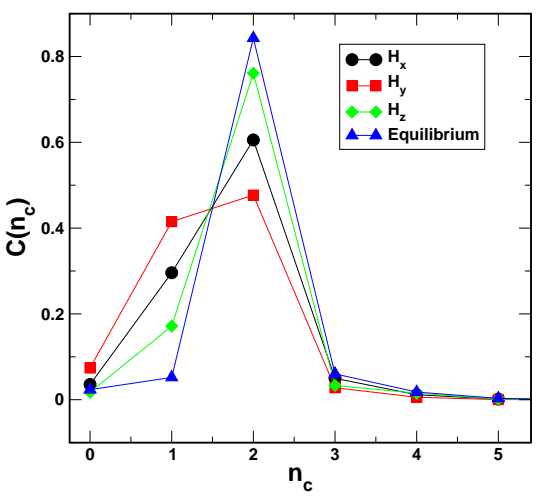

(b)

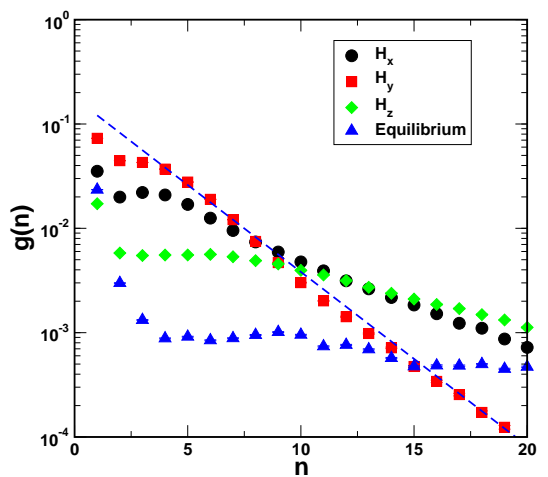

(c)

FIG. 15. For $\lambda \approx 6.17$ with applied field $H$ in shear thinning regime: (a) The configurational viscosity $\eta_{\text {conf }}$, the rotational viscosity $\eta_{\text {rot }}$ and the total viscosity $\eta_{\text {tot }}=\eta_{\text {conf }}+\eta_{\text {rot }}$ as a function of strength of the magnetic field $H$ are shown as circles, squares and diamond symbols, respectively. Top panel: Viscosities when the field is oriented in the flow direction, middle panel: when the field is oriented in the gradient of the flow direction, bottom panel: when the field is oriented in the vorticity direction. (b) The connectivity $C\left(n_{\mathrm{c}}\right)$ as a function of $n_{\mathrm{c}}$ for different orientations of magnetic field (c) Cluster size distribution $g(n)$ as a function of $n$ at different orientations of magnetic field. Exponential fit to the data is given as dashed line. All structural analysis are performed with a fixed magnetic field strength of $H=2.0$. Explanation is given in Sec. VB 3 .

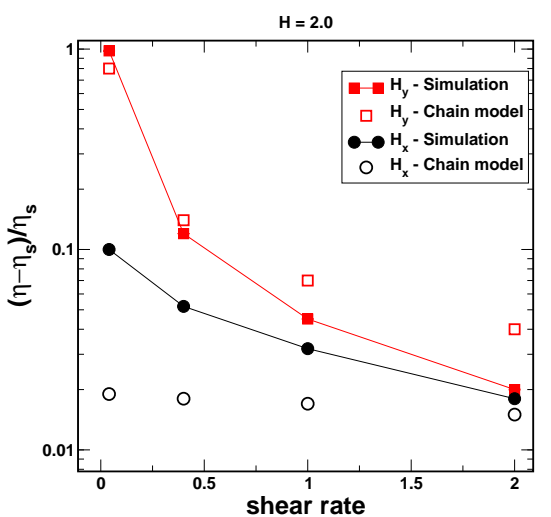

(a)
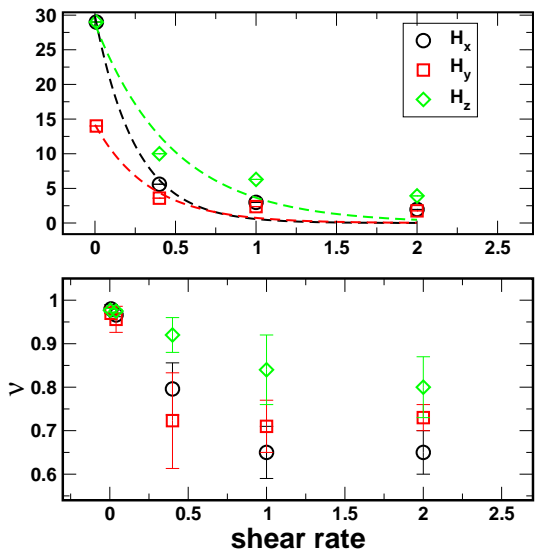

(b)

FIG. 16. For $\lambda \approx 6.17$ : (a) The total viscosities in the flow (circles) and gradient direction of flow (squares) from simulation (solid symbols) and the chain model prediction (open symbols computed by inputting value for chain size distribution from simulations) as a function of shear rate. (b) Top panel: Average cluster size as a function of shear rate. The exponential fit is shown as dashed lines. The rigidity exponent $\nu$ is shown for different orientation of magnetic field as a function of shear rate for the magnetic field strength $H=2.0$.

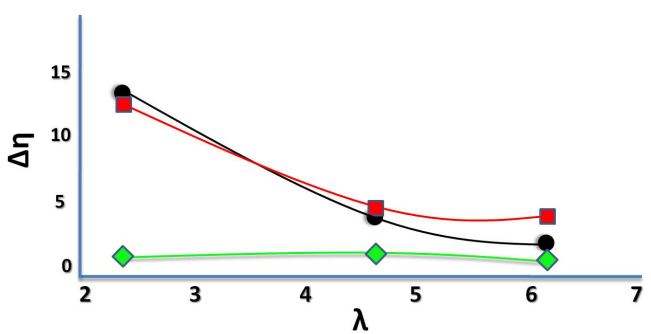

FIG. 17. A schematic of MVE in shear thinning regime for different dipolar interaction strengths. The MVE when the field is oriented in (1) the flow direction is shown as circles (2) gradient direction of the flow is shown as squares (3) the vorticity direction is denoted by diamonds. 


\section{MAGNETOVISCOUS EFFECT- COMPARISON WITH EXPERIMENTS}

According to the classic experiment by McTague [9], the viscosity of magnetic fluids is anisotropic and depends on the relative orientation of the magnetic field with respect to the flow. It is observed that the viscosity is larger when the magnetic field is oriented parallel to the flow direction compared to the case when the field is perpendicular to the flow. Here, we consider the Miesowicz viscosities in terms of coefficients parallel and perpendicular to a pipe flow. According to our procedure, the Miesowicz viscosity $\eta_{1}$ is parallel to the flow direction, thus $\eta_{\|}=\eta_{1}$. Other two Miesowicz viscosities $\eta_{2}$ in gradient direction of the flow and $\eta_{3}$ in the vorticity direction are perpendicular to the flow, thus $\eta_{\perp}=\frac{\eta_{2}+\eta_{3}}{2}[6]$. Thus the relative viscosity change is defined as

$$
\Delta \eta_{\| / \perp}=\frac{\eta_{\| / \perp}-\eta_{(H=0, \dot{\gamma})}}{\eta_{(H=0, \dot{\gamma})}} .
$$

We compare our simulation results with the experiment on the anisotropy of magnetoviscous effects in cobalt ferrofluids conducted by the group of Odenbach and coworkers [26]. As discussed in Sec. II, we parameterize our model by the magnetic volume fraction $\phi_{\mathrm{m}}=0.0021$ and dipolar interaction strength $\lambda=4.62$, which allows us to reproduce the magnetization curve rather well (see Fig. 1). We use the same dipolar interaction strength to study the MVE in the system. In addition to $\phi$ and $\sigma$, we need to fix a reference time scale in order to map the experimental shear rates to those in our simulations. The experimental data lack the information of clear transition from the Newtonian regime to the shear thinning regime (in fact there is no data available corresponding to the Newtonian regime). Thus, it is difficult to define a critical shear rate in experiments. But in our simulations, the transition from Newtonian regime to the shear thinning regime is well defined (see Fig. 2). Therefore, we define a characteristic time scale $\tau_{\text {shear }}$ which corresponds to the onset of shear thinning. Thus, dimensionless shear rate can be defined in terms of $\tau_{\text {shear }}$ as $D e=\dot{\gamma} \tau_{\text {shear }}$. For dipolar interaction strength $\lambda=4.62$, the characteristic time scale corresponding to the onset of shear thinning is $\tau_{\text {shear }} \approx 12 \tau_{\mathrm{B}}$. The shear thinning regime belongs to $1<D e<12$ and the first Newtonian regime is when $D e<1$. From our simulations we identify the regime where the experiments have been carried out. We find a range of shear rates which give the comparable results to the experimental shear rates. Using these details we find an approximate value of experimental relaxation time for the onset of shear thinning which is approximately $7( \pm 1)$ seconds. To check whether this value is reasonable, we matched the simulation and experimental shear rates for zero-field viscosity values and found that the onset of shear thinning overlaps approximately around $0.14 \pm 0.02 s^{-1}$. Available experimental data is in the shear thinning regime which is between $0.9-14 s^{-1}$ [26]. The dimensionless field $H$ used in the simulations
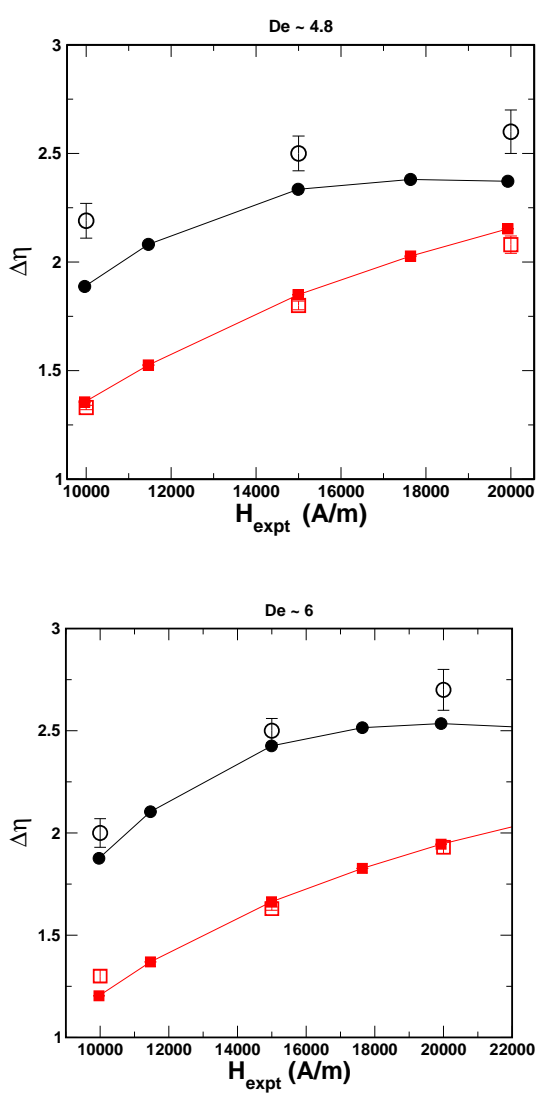

(b)
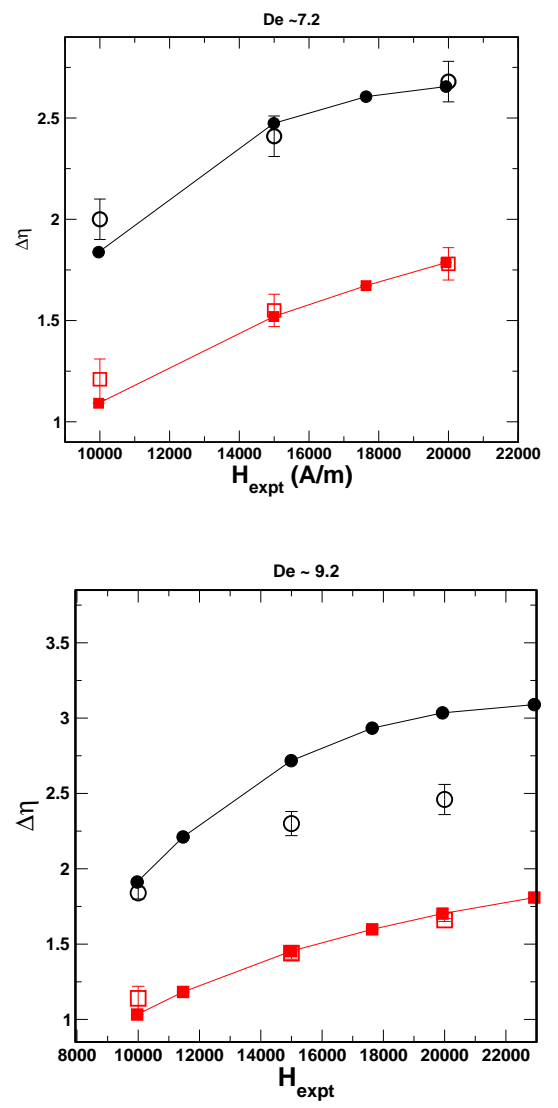

(d)

FIG. 18. The magnetoviscous effect $\Delta \eta$ for a range of dimensionless shear rates as a function of magnetic field strength (in real units) is computed from simulations (solid symbols) and compared with experiments (open symbols). $\Delta \eta_{\|}$(circles) is the magnetoviscous effect when the field is parallel to the flow and $\Delta \eta_{\perp}$ (squares) is the magnetoviscous effect when the field is perpendicular to the flow direction (see Eq. (11)). 


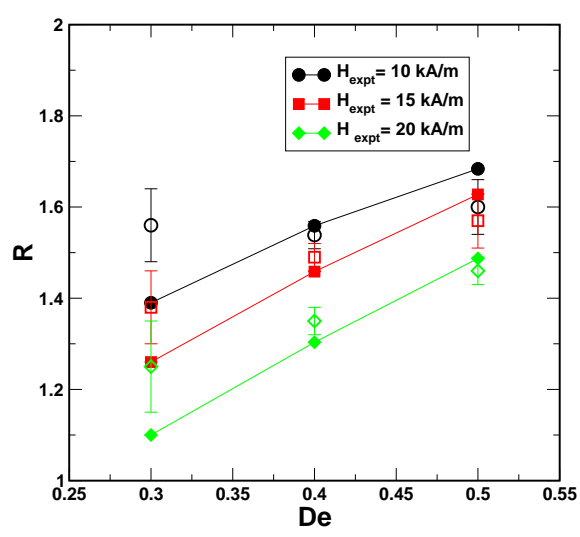

FIG. 19. The ratio $R$ of $\Delta \eta_{\|}$and $\Delta \eta_{\perp}$ as a function of dimensionless shear rate for different magnetic field strengths (solid and open symbols correspond to simulation and experiment, respectively).

is related to the experimentally applied field $H_{\text {expt }}$ by $H_{\text {expt }}=\frac{H}{\sqrt{4 \pi \mu_{0} \sigma^{3} /\left(k_{\mathrm{B}} T\right)}}=\frac{H}{1.1 \times 10^{-4}} \mathrm{~A} / \mathrm{m}$. We compare our simulation results for the same values of applied magnetic field strengths used in experiments, namely 10, 15 and $20 \mathrm{kA} / \mathrm{m}$.

We also compare the ratio of parallel and perpendicular MVE, given by $R=\frac{\Delta \eta_{\|}}{\Delta \eta_{\perp}}$. For a single particle rotation the ratio $R=2$ [32]. In Fig. 19, we show $R$ for a range of shear rates. All solid symbols corresponds to the simulation data and open symbols to experimental data.

Overall, we find that our simulation results are in semiquantitative agreement with experiments over a certain range of shear rates, but deviate for higher shear rates.

\section{SUMMARY AND DISCUSSIONS}

We have performed extensive non-equilibrium Langevin dynamics simulations to study the rheology of ferrofluids and the influence of micro-structure formation on rheology. We present here a concise summary of important results. We consider three values of dipolar interaction strengths corresponding to the weakly, moderately, and strongly interacting regime, respectively. There is hardly any chain formation in the weakly interacting regime and a massive structure formation is found in the strongly interacting regime. The intermediate case of moderate dipolar interaction corresponds most closely to the experimental situation [26]. There, we do find a certain degree of structure formation, as shown by the cluster analysis in Fig. 4.

In the zero field case, we observe a well defined Newtonian regime and a shear thinning regime in all dipolar interaction strengths. A prominent shear thinning is ob- served with increasing dipolar interaction strength. The onset of shear thinning shifts towards lower shear rate values with increasing $\lambda$. From the analysis of structural quantities, we find that with the increase in shear rate the micro-structures begin to rupture and form short chains, which can be directly correlated to the decrease in viscosity with increasing shear rates.

With the presence of an applied field, we have analysed changes in rheology in both Newtonian and shear thinning regime. The change in viscosity over the zero-field value (MVE) is found to have an interesting dependency on the orientation of the applied field and dipolar interaction strength. The MVE is larger in the case of intermediate interaction strength compared to weak and strong interaction strength cases when the field is applied along gradient of the flow direction. This is due to the prominent chain formation in intermediate interaction strength in the presence of an external field, which increases the contribution of both configurational and rotational viscosity. Even though there is an increase in absolute value of viscosity in the strong dipolar interaction strength, the relative change in viscosity from the zero-field viscosity is not as prominent as in the case of intermediate interaction strength. This is mainly due to the large value of zero-field viscosity that results from the presence of complex structures. The MVE in both Newtonian and shear thinning regime is influenced by the chain formation and chain rupturing. The analysis of cluster size distribution, connectivity and rigidity shows drastic structural changes (increase in the number of short chains and isolated particles) as the system moves from the Newtonian to shear thinning regime. This clearly explains the reduction in viscosity in the shear thinning regime.

The important point is that shear flow destroys the microstructures in the field-free case, but the combined action of a magnetic field and shear flow leads to the formation of larger structures (see Fig. 8) and corresponding viscosity changes.

We have compared viscosity changes obtained from simulations with the chain model (computed by inputting the cluster size distribution from simulations which is a pivotal quantity to the theoretical model) [19]. For weak dipolar interactions, we find discrepancies with the theoretical model prediction. Such discrepancies are to be expected since the theory is based on the formation of chains in the system, wherein no prominent structural formation occur in the weakly interacting regime. In the intermediate and strong interaction regimes, the chain model predictions matched well with most of the simulation data. Exceptions occur at high dipolar interaction strength for the viscosity change when the field is oriented in gradient direction of the flow, which needs to be understood better. We have also observed a stretched exponential decay behaviour of average cluster size with shear rate in the cases of intermediate and strong interaction strengths, which has not been reported in previous studies.

Another contribution to the magnetoviscous effect 
results from the shear-induced perturbation of shortrange correlation. The anisotropic pair correlations have been studied extensively in theory and in simulations $[7,20,29,33,34]$. The high-field anisotropy of pair correlation is observed in the study of Elfimova et al. [34]. In the weakly interacting case, the influence of short-range correlations on magnetoviscous effect has been studied theoretically and compared with simulations [20, 29]. For the moderately interacting case, the theoretical estimate leads only to a small contribution to the viscosity changes. In the simulations, the viscosity contributions of perturbed short-range correlations and small cluster formation are not easily distinguishable.

Finally we compare our results with the experiments on cobalt-based ferrofluids [26]. We parameterize our model in such a way that the simulations reproduce the equilibrium magnetisation curve measured experimentally. With the corresponding value of the dimensionless dipolar interaction strength $\lambda$, we mimic the experimental ferrofluids and perform non-equilibrium simulations to investigate the rheological properties. For a range of shear rates, we get comparable results. Since our simulations are performed at similar concentrations and dipolar interaction strengths as the experiments [26], we suggest that the same structural mechanisms are also relevant for the experimentally observed viscosity changes. For high shear rates, however, we observe our simulation results deviate from the experimental data. To further investigate these deviations, it would be interesting to incorporate polydispersity effects, employ a more detailed modelling of repulsive interactions and include hydrodynamic interactions in the model.

\section{ACKNOWLEDGMENTS}

We are grateful to H. C. Öttinger at ETH Zürich for his kind support. We acknowledge S. Odenbach, J. Linke and D. Borin at Technical University, Dresden for valuable discussions and for providing experimental data. We are grateful to the Swiss National Science Foundation (SNSF) for funding this project 20PA21E-129506: "Experiment and Computer simulation of the anisotropy of magnetoviscous effect".
[1] S. Odenbach. Magnetoviscous Effects in Ferrofluids, volume 71 of Lecture Notes in Phys. Springer, Berlin, 2002.

[2] R. E. Rosensweig, R. Kaiser, and G. Miskolczy. Viscosity of magnetic fluid in a magnetic field. J. Colloid Interface Sci., 29(4):680, 1969.

[3] A. Cebers. Simulation of the magnetic rheology of a dilute suspension of ellipsoidal particles in a numerical experiment. Magnetohydrodynamics, 20:349-354, 1984.

[4] R. E. Rosensweig. Ferrohydrodynamics. Cambridge University Press, Cambridge, 1985.

[5] A. Satoh, R. W. Chantrell, G. N. Coverdale, and S. Kamiyama. Stokes dynamics simulations of ferromagnetic colloidal dispersions in a simple shear flow. J. Colloid Interface Sci., 203:233-248, 1998.

[6] M. Kröger, P. Ilg, and S. Hess. Magnetoviscous model fluids. J. Phys.: Condens. Matter, 15:S1403-1423, 2003.

[7] P. Ilg and S. Odenbach. Ferrofluid structure and rheology. In S. Odenbach, editor, Colloidal Magnetic Fluids: Basics, Development and Applications of Ferrofluids, volume 763 of Lecture Notes in Phys. Springer, Berlin, 2008.

[8] D Soto-Aquino and C Rinaldi. Magnetoviscosity in dilute ferrofluids from rotational brownian dynamics simulations. Phys. Rev. E, 82(4):046310, 2010.

[9] J. P. McTague. Magnetoviscosity of magnetic colloids. J. Chem. Phys., 51:133-136, 1969.

[10] M. I. Shliomis. Effective viscosity of magnetic suspensions. Sov. Phys. JETP, 34(6):1291-1294, 1972.

[11] A. Grants, A. Irbitis, G. Kronkalns, and M. M. Maiorov. Rheological properties of magnetite magnetic fluid. $J$. Magn. Magn. Mater., 85:129-132, 1990.

[12] D. Borin, A. Zubarev, D. Chirikov, R. Müller, and S. Odenbach. Ferrofluid with clustered iron nanoparticles: Slow relaxation of rheological properties under joint action of shear flow and magnetic field. J. Magn. Magn.
Mater., 323(10):1273-1277, 2011.

[13] L. M Pop and S. Odenbach. Capillary viscosimetry on ferrofluids. J. Phys.: Condens. Matter, 20(20):204139, 2008.

[14] R. Patel. Ferrohydrodynamic evaluation of rotational viscosity and relaxation in certain ferrofluids. Phys. Rev. E, 86(1):016324, 2012.

[15] O. Ambacher, S. Odenbach, and K. Stierstadt. Rotational viscosity in ferrofluids. Z. Phys. B. Cond. Mat., 86:29-32, 1992 .

[16] S. Odenbach and H. Störk. Shear dependence of field induced contributions to the viscosity of magnetic fluids at low shear rates. J. Magn. Magn. Mater., 183:188-194, 1998.

[17] S. Odenbach, T. Rylewicz, and M. Heyen. A rheometer dedicated for the investigation of viscoelastic effects in commercial magnetic fluids. J. Magn. Magn. Mater., 201:155-158, 1999

[18] H. W. Müller and M. Liu. Structure of ferrofluid dynamics. Phys. Rev. E, 64:061405, 2001.

[19] A. Yu. Zubarev and L. Yu. Iskakova. Effect of chainlike aggregates on dynamical properties of magnetic liquids. Phys. Rev. E, 61(5):5415-5421, 2000.

[20] P. Ilg, M. Kröger, and S. Hess. Magnetoviscosity of semi-dilute ferrofluids and the role of dipolar interactions: Comparison of molecular simulations and dynamical mean-field theory. Phys. Rev. E, 71:031205, 2005.

[21] P. Ilg, M. Kröger, and S. Hess. Anisotropy of the magnetoviscous effect in ferrofluids. Phys. Rev. E, 71:051201, 2005.

[22] Z. Wang, C. Holm, and H. W. Müller. Molecular dynamics study on the equilibrium magnetization properties and structure of ferrofluids. Phys. Rev. E, 66:021405, 2002 . 
[23] P. Ilg and M. Kröger. Anisotropic self-diffusion in ferrofluids studied via brownian dynamics simulations. Phys. Rev. E, 72(3):031504, 2005.

[24] A. Sreekumari and P. Ilg. Slow relaxation in structureforming ferrofluids. Phys. Rev. E, 88(4):042315, 2013.

[25] M. P. Allen and D. J. Tildesley. Computer Simulation of Liquids. Oxford University Press, Oxford, 1987.

[26] M. Gerth-Noritzsch, D. Yu Borin, and S. Odenbach. Anisotropy of the magnetoviscous effect in ferrofluids containing nanoparticles exhibiting magnetic dipole interaction. J. Phys.: Condens. Matter, 23:346002, 2011.

[27] M. I. Shliomis. Ferrohydrodynamics: Retrospective and issues. In S. Odenbach, editor, Ferrofluids. Magnetically Controllable Fluids and Their Applications, number 594 in Lecture Notes in Physics, pages 85-111. Springer, Berlin, 2002.

[28] S. Hess. Flow properties and structure of anisotropic fluids studied by non-equilibrium molecular dynamics, and flow properties of other complex fluids: polymeric liquids, ferro-fluids and magneto-rheological fluids. In P. Parisi and C. Zannoni, editors, Advances in the computer simulation of liquid-crystals, pages 189-233. Kluwer, Dor- drecht, 2000.

[29] P. Ilg and S. Hess. Nonequilibrium dynamics and magnetoviscosity of moderately concentrated magnetic liquids: A dynamic mean-field study. Z. Naturforsch., 58a:589$600,2003$.

[30] A. Yu. Zubarev, J. Fleischer, and S. Odenbach. Towards a theory of dynamical properties of polydisperse magnetic fluids: Effect of chain-like aggregates. Physica A, 358:475-491, 2005.

[31] P. Ilg, E. Coquelle, and S. Hess. Structure and rheology of ferrofluids: simulation results and kinetic models. J. Phys.: Condens. Matter, 18:S2757-S2770, 2006.

[32] P. Ilg and M. Kröger. Magnetization dynamics, rheology, and an effective description of ferromagnetic units in dilute suspension. Phys. Rev. E, 66:021501, 2002. Erratum: Phys. Rev. E, 67:049901, 2003.

[33] P. Ilg, M. Kröger, and S. Hess. Structure and rheology of model-ferrofluids under shear flow. J. Magn. Magn. Mater., 289C:325-327, 2005.

[34] E. A. Elfimova, A. O. Ivanov, and P. J. Camp. Theory and simulation of anisotropic pair correlations in ferrofluids in magnetic fields. J. Chem. Phys., 136(19):194502, 2012 . 